\title{
Charge transport in non-polar and semi-polar III-V nitride heterostructures
}

\author{
Aniruddha Konar, Amit Verma, Tian Fang, Pei Zhao, Raj Jana \\ and Debdeep Jena
}

Department of Electrical Engineering, University of Notre Dame, IN 46556, USA

E-mail: akonar@nd.edu

Received 10 October 2011, in final form 13 December 2011

Published 19 January 2012

Online at stacks.iop.org/SST/27/024018

\begin{abstract}
Compared to the intense research focus on the optical properties, the transport properties in non-polar and semi-polar III-nitride semiconductors remain relatively unexplored to date. The purpose of this paper is to discuss charge-transport properties in non-polar and semi-polar orientations of $\mathrm{GaN}$ in a comparative fashion to what is known for transport in polar orientations. A comprehensive approach is adopted, starting from an investigation of the differences in the electronic bandstructure along different polar orientations of GaN. The polarization fields along various orientations are then discussed, followed by the low-field electron and hole mobilities. A number of scattering mechanisms that are specific to non-polar and semi-polar GaN heterostructures are identified, and their effects are evaluated. Many of these scattering mechanisms originate due to the coupling of polarization with disorder and defects in various incarnations depending on the crystal orientation. The effect of polarization orientation on carrier injection into quantum-well light-emitting diodes is discussed. This paper ends with a discussion of orientation-dependent high-field charge-transport properties including velocity saturation, instabilities and tunneling transport. Possible open problems and opportunities are also discussed.
\end{abstract}

(Some figures may appear in colour only in the online journal)

\section{Introduction}

The III-nitride semiconductors and related compounds have attracted immense attention for optoelectronic devices [1] covering a wide range of the electromagnetic spectrum. They have also been found well suited for high-speed, hightemperature electronic devices [2]. The demonstration of high electron mobility transistors (HEMTs) using AlGaNGaN heterojunctions [3] paved the way for applications in high-speed and high-power electronics. The wide span of the bandgaps of the III-V nitride semiconductor family, ranging from infrared (indium nitride $(\mathrm{InN}), E_{g}=0.7 \mathrm{eV}$ ) to deep ultraviolet (aluminum nitride $(\mathrm{AlN}), E_{g}=6.2$ ) makes them excellent candidates for applications in shortwavelength lasers and in light-emitting diodes (LEDs). Since the demonstration of high-efficiency InGaN blue LEDs by Nakamura et al [4], nitrides have found their way into many commercial optoelectronics device applications.
Traditionally, nitride semiconductors are epitaxially grown in the $c$-plane of the wurtzite (WZ) crystal structure as shown in figure $1(a)$. The uniaxial $c$-direction perpendicular to the $c$-plane constitutes the polar orientation of $\mathrm{GaN}$ (and $\mathrm{InN}$ and $\mathrm{AlN}$ ). For $c$-plane nitrides, the built-in polarization field is advantageous for the formation of two-dimensional electron gases (2DEG) (see figure $1(d)$ ) for transistor applications [5]. In quantum well (QW)-based optical devices, this polarization field reduces the overlap of electron-hole wavefunctions, reducing the oscillator strength [6] of radiative transitions. Moreover, $c$-plane-based enhancement-mode transistors suffer from low threshold voltages [7] $\left(V_{\text {th }} \sim 1 \mathrm{~V}\right)$, impeding their applications in circuit operations. A potential way to eliminate these effects is to grow nitrides in semi-polar [8] and non-polar directions. A non-polar plane is a crystal plane perpendicular to the $c$-plane, and the polarization vector lies in the plane of growth. On the other hand, a semi-polar plane intersects the $c$-plane at an angle as shown in figure 1(b). The common non-polar 


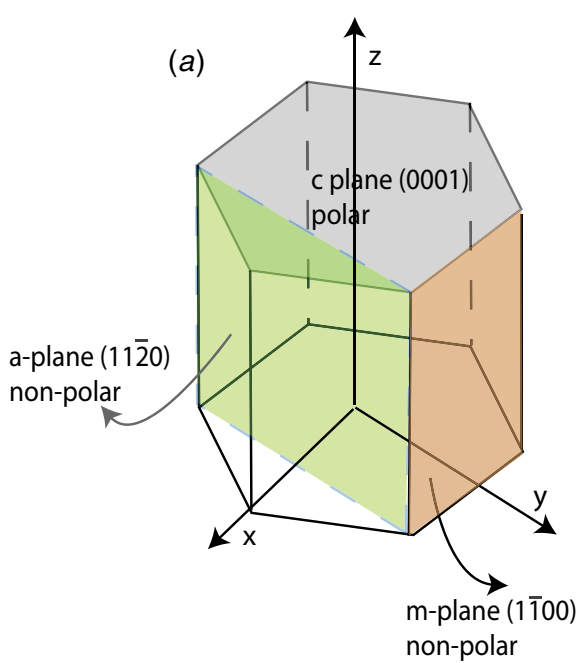

(c)

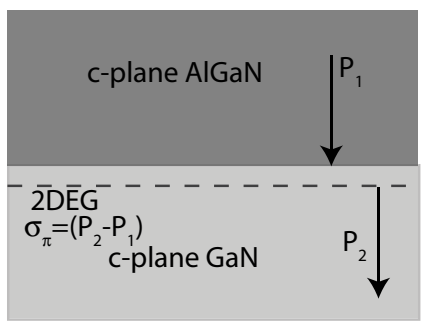

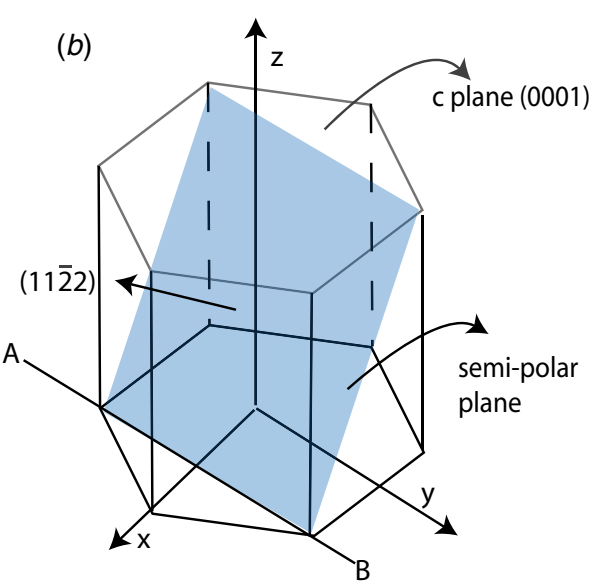

$(d)$

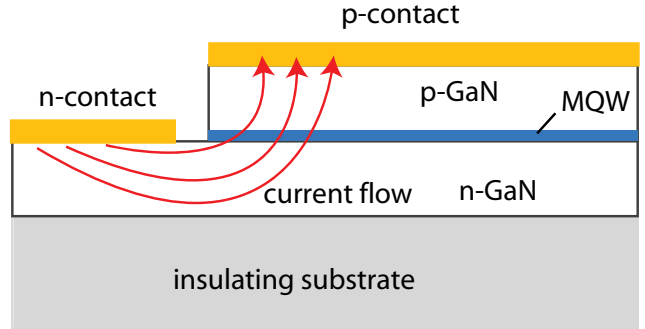

Figure 1. Schematic diagram of $(a)$ polar and non-polar crystal planes, $(b)$ semi-polar crystal plane passing through the line AB, (c) GaN/AlGaN heterojunction and polarization-induced 2DEG and $(d)$ typical $\mathrm{p}-\mathrm{n}$ junction LED device showing the path of current flow.

planes of current interest are the $m$-plane $\left(\begin{array}{lll}1 & 1 & 0\end{array}\right)$ [1, 10-12], and the $a$-plane $(11 \overline{2} 0)$ [1, 10-13] (see figure 1). Semi-polar

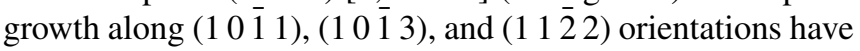
been achieved [14].

In-plane charge transport in traditional $c$-plane nitride devices has been investigated extensively, both theoretically and experimentally $[5,9]$. Compared to the intense research focus on the optical properties, the transport properties in nonpolar and semi-polar nitrides remain relatively unexplored to date. The purpose of this paper is to discuss charge-transport properties in non-polar and semi-polar orientations of $\mathrm{GaN}$ in a comparative fashion to what is known for transport in polar orientations. To do so, a comprehensive approach is taken. The differences in the electronic bandstructure along different polar orientations of $\mathrm{GaN}$ are first explored in a $k \cdot p$ formalism. The polarization fields along various orientations are then discussed. Then, low-field electron and hole mobilities are discussed; a number of scattering mechanisms that are specific to non-polar and semi-polar GaN heterostructures are identified, and their effects are evaluated. The effect of polarization orientation on carrier injection into QW LEDs is discussed. Finally, a discussion of orientation-dependent highfield charge-transport properties including velocity saturation, instabilities, and tunneling transport is included. Possible open problems and opportunities are also discussed. Since transport properties hinge on the nature of the electronic energy spectrum, we begin the discussion with the bandstructure of $\mathrm{GaN}$ along various crystal orientations.

\section{Electronic bandstructure of polar, semi-polar and non-polar bulk nitrides}

The effective mass $\left(m^{\star}\right)$ of carriers is one of the fundamental parameters in the context of charge transport in solids. For lowfield transport, the Fermi level usually lies close to the band edges, and carriers populate low-energy states. The effective mass of electrons and holes near the band-minima point are the important parameters to determine the transport quantities in this regime. At high electric fields, higher energy regions of the energy dispersion play an important role in determining the saturation velocity as well as microwave oscillations (the Gunn effect). The $c$-plane has the full WZ symmetry and the in-plane strain components are isotropic. As a result, the motion of carriers in the $c$-plane is isotropic, as embodied in an isotropic effective mass. Semi-polar and non-polar planes have lower symmetries and the electronic $[29,30]$ and optical properties $[15,16]$ are direction dependent due to anisotropic strain components and polarization fields. In this section, we investigate the band structure of III-V nitrides for an arbitrary growth direction using the $k \cdot p$ theory originally developed by Kane [17] and then improved by Chuang et al [18] using Lowdin's [19] perturbation method. We compare the bandedge effective masses of polar, semi-polar and non-polar GaN calculated from the $k \cdot p$ theory with the values obtained from density functional theory (DFT). 
Table 1. Parameters for GaN used in the $k \cdot p$ theory.

\begin{tabular}{|c|c|c|c|}
\hline Parameter & $\mathrm{GaN}$ & Parameter & $\mathrm{GaN}$ \\
\hline Lattice constant $^{\mathrm{a}}(\AA)$ & & Deformation potentials $(\mathrm{eV})^{\mathrm{b}}$ & \\
\hline$a$ & 3.189 & $D_{1}$ & -3.6 \\
\hline$c$ & 5.185 & $D_{2}$ & 1.7 \\
\hline Energy Parameters $^{\mathrm{a}}$ & & $D_{3}$ & 5.2 \\
\hline$E_{g}(\mathrm{eV})$ & 3.40 & $D_{4}$ & -2.7 \\
\hline$\Delta_{\mathrm{cr}}=\Delta_{1}(\mathrm{meV})$ & 22.0 & $D_{5}$ & -2.8 \\
\hline$\Delta_{\mathrm{so}}=3 \Delta_{2}(\mathrm{meV})$ & 17.0 & $D_{6}$ & -4.3 \\
\hline$\Delta_{2}=\Delta_{\mathrm{so}} / 3(\mathrm{meV})$ & 5.66 & Elastic stiffness constant ${ }^{\mathrm{b}}\left(10^{10} \mathrm{~N} \mathrm{~m}^{-2}\right)$ & \\
\hline Conduction-band effective masses ${ }^{b}$ & & $C_{11}$ & 39.0 \\
\hline$m_{e}^{\star}(\| c)$ & $0.21 m_{0}$ & $C_{12}$ & 14.5 \\
\hline$m_{e}^{\star}(\perp c)$ & $0.20 m_{0}$ & $C_{13}$ & 10.6 \\
\hline Valence-band effective-mass parameters ${ }^{\mathrm{a}}$ & & $C_{33}$ & 39.8 \\
\hline$A_{1}$ & -6.56 & $C_{44}$ & 10.5 \\
\hline$A_{2}$ & -0.91 & $P_{\mathrm{sp}}^{\mathrm{c}}\left(\mathrm{Cm}^{-2}\right)$ & -0.029 \\
\hline$A_{3}$ & 5.65 & Piezoelectric constant ${ }^{\mathrm{c}}\left(\mathrm{C} \mathrm{m}^{-2}\right)$ & \\
\hline$A_{4}$ & -2.83 & $e_{15}$ & -0.22 \\
\hline$A_{5}$ & -3.13 & $e_{31}$ & -0.34 \\
\hline$A_{6}$ & -4.86 & $e_{33}$ & 0.67 \\
\hline
\end{tabular}

\subsection{Valence-band and Conduction-band Hamiltonian}

The valence bands of WZ crystals retain properties of atomic $\mathrm{P}$ orbitals, similar to other III-V semiconductors. Representing the three atomic P-orbital component states as $|X\rangle,|Y\rangle$ and $|Z\rangle$, we define six basis functions as their linear combinations: $(-1 / \sqrt{2})|(X+\mathrm{i} Y) \uparrow\rangle,(-1 / \sqrt{2})|(X+\mathrm{i} Y) \downarrow\rangle,|Z \uparrow\rangle,|Z \downarrow\rangle$, $(-1 / \sqrt{2})|(X-\mathrm{i} Y) \uparrow\rangle$, and $(-1 / \sqrt{2})|(X-\mathrm{i} Y) \downarrow\rangle$. Here, $\uparrow(\downarrow)$ stands for the spin-up (down) states. Using this basis set, the $6 \times 6$ valence-band Hamiltonian for $\left(\begin{array}{llll}0 & 0 & 0 & 1\end{array}\right)$-oriented bulk WZ nitrides can be written using degenerate perturbation theory as $[18,9]$

$$
\begin{aligned}
\boldsymbol{H}_{v}\left(k_{x}, k_{y}, k_{z}, \epsilon\right) & \\
= & \left(\begin{array}{cccccc}
F & -K^{\star} & -H^{\star} & 0 & 0 & 0 \\
-K & G & H & 0 & 0 & \Delta \\
-H & -H^{\star} & \Lambda & 0 & \Delta & 0 \\
0 & 0 & 0 & F & -K & H \\
0 & 0 & \Delta & -K^{\star} & G & -H^{\star} \\
0 & \Delta & 0 & H^{\star} & -H & \Lambda
\end{array}\right),
\end{aligned}
$$

where

$$
\begin{aligned}
& F=\Delta_{\text {cr }}+\frac{\Delta_{\text {so }}}{3}+\Theta, \\
& G=\Delta_{\text {cr }}-\frac{\Delta_{\text {so }}}{3}+\Lambda+\Theta, \\
& \lambda=\frac{\hbar^{2}}{2 m_{0}}\left[A_{1} k_{z}^{2}+A_{2}\left(k_{x}^{2}+k_{y}^{2}\right)\right]+\Lambda_{\epsilon}, \\
& \Theta=\frac{\hbar^{2}}{2 m_{0}}\left[A_{3} k_{z}^{2}+A_{4}\left(k_{x}^{2}+k_{y}^{2}\right)\right]+\Theta_{\epsilon}, \\
& K=\frac{\hbar^{2}}{2 m_{0}} A_{5}\left(k_{x}+\mathrm{i} k_{y}\right)^{2}+D_{5}\left(\epsilon_{x x}-\epsilon_{y y}\right), \\
& H=\frac{\hbar^{2}}{2 m_{0}} A_{6}\left(k_{x}+\mathrm{i} k_{y}\right) k_{z}+D_{6} \epsilon_{z z}, \\
& \Lambda_{\epsilon}=D_{1} \epsilon_{z z}+D_{2}\left(\epsilon_{x x}+\epsilon_{y y}\right), \\
& \Theta_{\epsilon}=D_{3} \epsilon_{z z}+D_{4}\left(\epsilon_{x x}+\epsilon_{y y}\right),
\end{aligned}
$$

$$
\Delta=\frac{\sqrt{2}}{3} \Delta_{\text {so }}
$$

Here, $\hbar$ is the reduced Planck's constant, $m_{0}$ is the bare electron mass, $k_{i}(i=x, y, z)$ is the $i$ th component of the wave vector $\boldsymbol{k}$, and $\epsilon_{i j}$ denotes the different strain components. The valence-band effective-mass parameters $A_{i}$, the deformation potentials $D_{i}(i=1-6)$, the splitting energies $\Delta_{\text {cr }}$ and $\Delta_{\text {so }}$, and all other physical parameters appearing in the above formulation have their usual meanings and are tabulated in table 1 .

The conduction band is $S$-like and the basis set is simpler: $|S \uparrow\rangle$ and $|S \downarrow\rangle$. The bulk Hamiltonian for the conduction band of a $c$-plane WZ crystal has the form

$$
\begin{array}{r}
\boldsymbol{H}_{c}\left(k_{x}, k_{y}, k_{z}, \epsilon\right)=E_{c}+\frac{\hbar^{2}\left(k_{x}^{2}+k_{y}^{2}\right)}{2 m_{\|}} \\
+\frac{\hbar^{2} k_{z}^{2}}{2 m_{\perp}}+a_{c 1} \epsilon_{z z}+a_{c 2}\left(\epsilon_{x x}+\epsilon_{y y}\right),
\end{array}
$$

where $m_{\|}\left(m_{\perp}\right)$ is the parallel (perpendicular) effective mass to the $c$-plane, $E_{c}=E_{g}+\Delta_{\mathrm{cr}}+\frac{\Delta_{\mathrm{so}}}{3}$, and $a_{c 1}$ and $a_{c 2}$ are the conduction-band deformation potentials.

The above Hamiltonians are constructed using a coordinate system $(x, y, z)$ suitable for describing $c$-plane growth as shown in figure 2(a). To describe the bands in non$c$-plane nitrides, one needs to rotate the coordinate system and go from the unprimed to the primed coordinates $\left(x^{\prime}, y^{\prime}, z^{\prime}\right)$ [20-22] as shown in figure 2. We rotate the coordinate system in such a way that $z^{\prime}$ points along the growth direction. With such a condition, any non-polar or semi-polar plane can be described by two rotation angles $\theta$ and $\phi$, respectively. For example, for the $c$-plane, $\theta=0$ and $\phi=0$. For the $a$-plane, $\theta=\pi / 2$ and $\phi=0$, for the $m$-plane, $\theta=\pi / 2$ and $\phi=\pi / 6$, and so on. In this work, we consider semi-polar and non-polar planes passing through the line $A B(\phi=0)$. For such planes, 

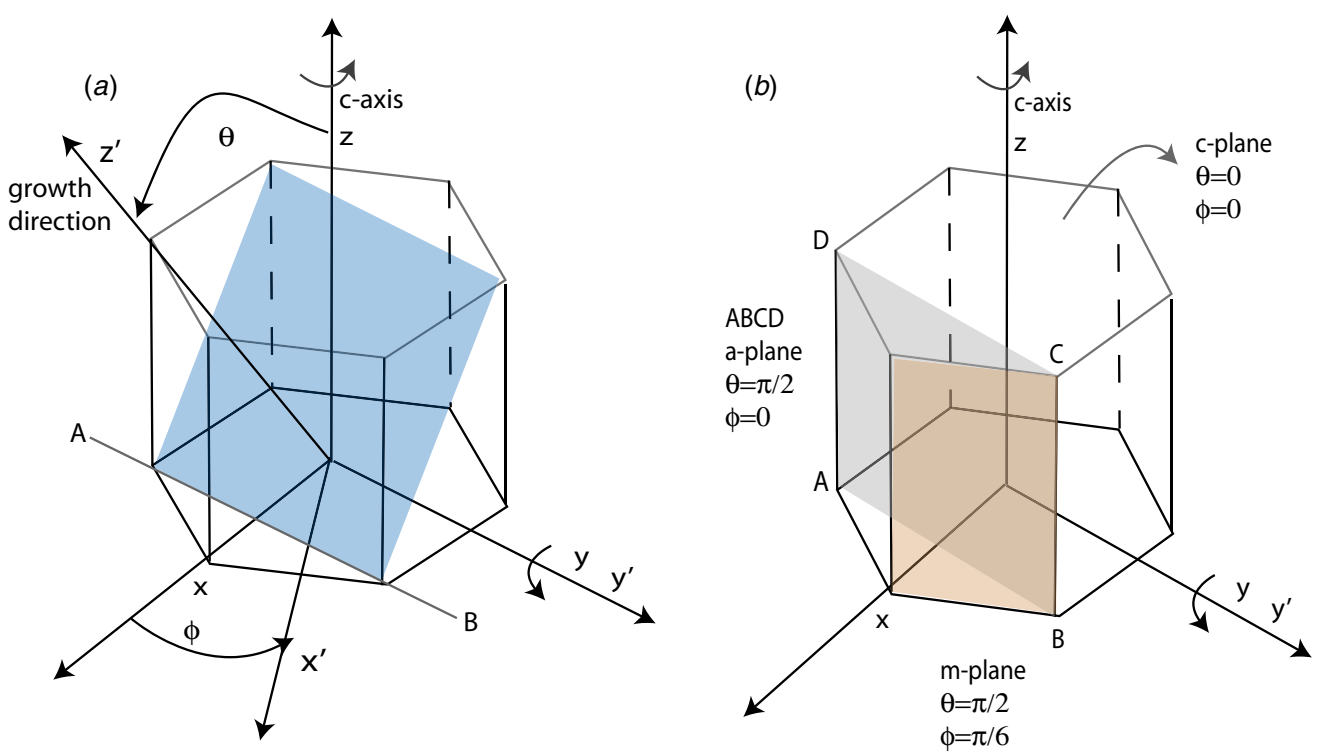

Figure 2. (a) Coordinate systems used in this work and (b) polar angles of various non-polar and polar planes.

the wave vector in the primed coordinate is given by $\boldsymbol{k}^{\prime}=\mathcal{U} \boldsymbol{k}$, where the rotation matrix $\mathcal{U}$ is

$$
\mathcal{U}=\left(\begin{array}{ccc}
\cos \theta & 0 & \sin \theta \\
0 & 1 & 0 \\
-\sin \theta & 0 & \cos \theta
\end{array}\right) .
$$

The strain components $\epsilon_{i j}$ in the $(x, y, z)$ coordinates for a general crystal orientation are calculated following the procedure outlined in $[20,23]$ :

$$
\begin{aligned}
& \epsilon_{x x}=\epsilon_{x x}^{(0)}+\epsilon_{x z} \tan \theta, \\
& \epsilon_{y y}=\epsilon_{x x}^{(0)}, \\
& \epsilon_{z z}=\epsilon_{z z}^{(0)}+\epsilon_{x z} \cot \theta,
\end{aligned}
$$

where $\epsilon_{x x}^{(0)}=\left(a_{s}-a_{e}\right) / a_{e}, \epsilon_{z z}^{(0)}=\left(c_{s}-a_{s}\right) / a_{e}$, and $c_{i j}$ denotes the elastic constants of the material. All other strain components are zero, and $a_{e}\left(a_{s}\right)$ and $c_{e}\left(c_{s}\right)$ are the equilibrium lattice constants of the bulk nitrides. The $x z$ component of the strain tensor is given by

$\epsilon_{x z}$

$=-\frac{\left(\left[c_{11} \epsilon_{x x}^{(0)}+c_{12} \epsilon_{x x}^{(0)}+c_{13} \epsilon_{z z}^{(0)}\right] \sin ^{2} \theta+\left[2 c_{13} \epsilon_{x x}^{(0)}+c_{33} \epsilon_{z z}^{(0)}\right] \cos ^{2} \theta\right) \sin \theta \cos \theta}{c_{11} \sin ^{4} \theta+2\left(c_{13}+2 c_{44}\right) \sin ^{2} \theta \cos ^{2} \theta+c_{33} \cos ^{4} \theta}$

Using the above strain components and transforming the wave vector using the relation $\boldsymbol{k}=\mathcal{U}^{T} \boldsymbol{k}^{\prime}$ (where $T$ stands for transpose), the electronic bandstructures of bulk nitrides are easily obtained by diagonalizing the Hamiltonian matrix in equation (1). Figures 3(a)-(c) show the conduction- and valence-band structures of unstrained bulk GaN with inplane wave vectors $\left(k_{x}^{\prime}, k_{y}^{\prime}\right)$ for polar, semi-polar and nonpolar growth directions. The semi-polar plane considered here is the $(11 \overline{2} 2)$ plane corresponding to $\theta \approx 58^{\circ}$ with the $c$-axis. The conduction bands are symmetric and are independent of growth directions. The valence bands show significant differences for different crystal planes. The

\begin{tabular}{|c|c|c|c|}
\hline $\begin{array}{l}\text { Growth } \\
\text { plane }\end{array}$ & $\begin{array}{l}\text { Growth } \\
\text { direction }\end{array}$ & $m_{x^{\prime} x^{\prime}}^{h h} / m_{0}$ & $m_{y^{\prime} y^{\prime}}^{h h} / m_{0}$ \\
\hline$c$-plane & $\left(\begin{array}{llll}0 & 0 & 0 & 1\end{array}\right)$ & $0.37(\Gamma \rightarrow \mathrm{K})$ & $0.37(\Gamma \rightarrow \mathrm{M})$ \\
\hline Semi-polar & 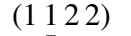 & 0.72 & $0.37(\Gamma \rightarrow \mathrm{M})$ \\
\hline$a$-plane & $(1 \overline{1} 20)$ & $1.1(\Gamma \rightarrow \mathrm{A})$ & $0.37(\Gamma \rightarrow \mathrm{M})$ \\
\hline$m$-plane & $(1 \overline{1} 00)$ & $0.37(\Gamma \rightarrow \mathrm{K})$ & $1.1(\Gamma \rightarrow \mathrm{A})$ \\
\hline
\end{tabular}
$c$-plane has the full WZ symmetry and the valence bands are
Table 2. Heavy-hole effective mass of polar, semi-polar and non-polar $\mathrm{GaN}$

isotropic with in-plane wave vectors. The semi-polar and and non-polar planes lack the full WZ symmetry and the valence bands show in-plane anisotropy. The energy contour plots in figures $3(d)-(f)$ of the top valence band (heavy hole) capture this anisotropy. The anisotropic valence bands lead to anisotropic hole effective mass at the band edge which in effect leads to directionally dependent transport quantities. The band-edge heavy-hole effective masses $\left(m_{x^{\prime} x^{\prime}}^{h h}\right.$, and $\left.m_{y^{\prime} y^{\prime}}^{h h}\right)$ and along two in-plane principal axes $\left(x^{\prime}, y^{\prime}\right)$ are tabulated in table 2. We note here that the above band structure is a lowenergy spectrum of electrons necessary for understanding lowfield transport. We defer the discussion of the higher energy regions in the Brillouin zone away from the $\Gamma$ point to later in the document to go with high-field transport. Armed with these underpinnings of the electronic band structure of $\mathrm{GaN}$ along various crystal orientations, we proceed with a discussion of low-field charge transport.

\section{Charge transport in non-polar and semi-polar nitrides}

In this section, we review the low-field charge transport in nonpolar and semi-polar bulk nitrides as well as in heterojunction QWs. The charge transport is assumed to occur in the growth plane perpendicular to the growth direction, as is the case in field-effect transistors, or in lateral carrier spreading in optical 


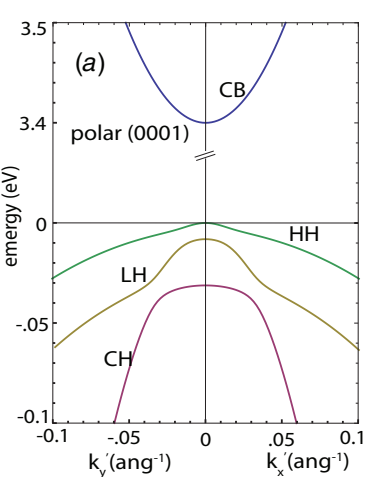

$(d)$

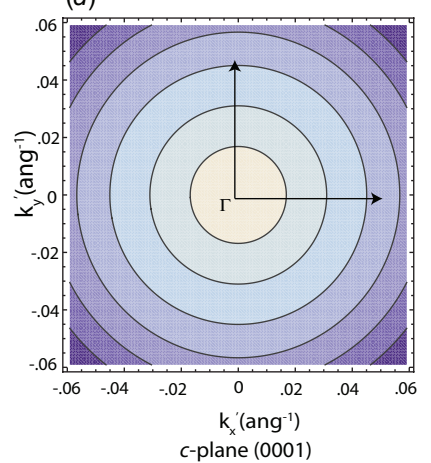

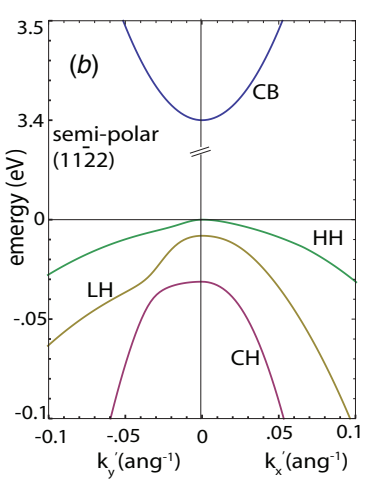

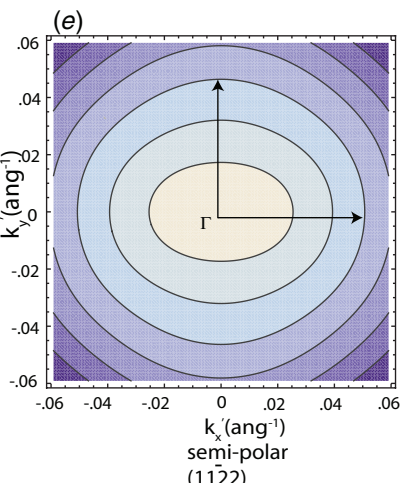

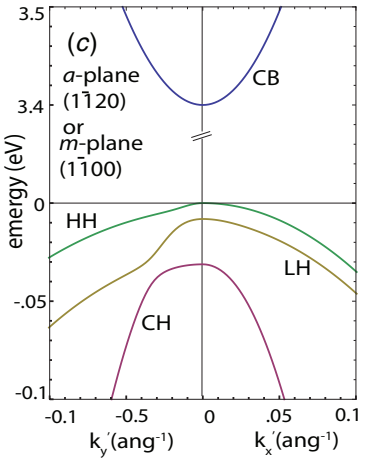

$(f)$

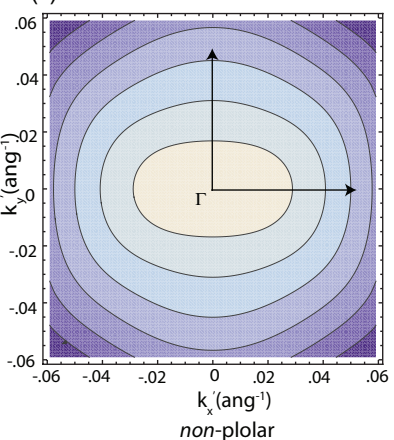

Figure 3. Electronic bandstructure with in-plane wave vectors for $(a)$ polar, $(b)$ semi-polar and $(c)$ non-polar GaN. The energy reference is taken at the top of the topmost valence band. $(d)-(f)$ Constant energy contour plots of the topmost valence band for polar, semi-polar and non-polar GaN, highlighting the asymmetry and warping. The energy spacing for each contour plots is 3 meV, starting from zero at the center.

devices. The basic scattering mechanisms of non-polar and semi-polar planes are similar to those of $c$-plane transport. The differences arise from the existence of non-vanishing polarization in the transport plane of non-polar and semipolar nitrides. The coupling of this in-plane polarization with disorder and defects leads to transport quantities different from polar nitrides. Following [24, 25], we discuss the experimental and theoretical reports of charge transport in the non-polar $m$-plane or $a$-plane. Transport in semi-polar planes is also outlined.

\subsection{Stacking fault mediated tunneling transport in non-polar bulk GaN}

Heteroepitaxial, non-polar and semi-polar nitride films grown on lattice mismatched substrates can contain $n_{\mathrm{SF}} \sim 1-$ $10 \times 10^{5} / \mathrm{cm}$ basal stacking faults (BSFs) parallel to the [0 $\left.\begin{array}{lll}0 & 0 & 1\end{array}\right]$ direction [26-28]. Directionally dependent transport measurements in structures infected with stacking faults in non-polar nitride films show a strong anisotropy in the mobility for both electron and hole transport. The mobility parallel to the [0 $\left.\begin{array}{lll}0 & 0 & 1\end{array}\right]$ direction is significantly lower relative to that in the in-plane [1 $11 \overline{2} 0$ ] directions [29-31]. This transport anisotropy can be explained using the methodology developed for understanding carrier transport properties across a BSF in the presence of polarization [25], as discussed now.

3.1.1. Energy band diagram across a BSF. A BSF is a thin zincblende (ZB) layer (up to three monolayers thick) smoothly inserted in the WZ matrix without broken bonds as shown in figure $4(a)$. The built-in polarization difference between $\mathrm{ZB}$ and WZ structures will result in bound sheet charges $\pm \sigma_{\pi}$ at each interface of the BSF layer. The band edge will consequently bend near the BSF. Figure 4(b) shows a typical conduction band diagram around a BSF for an n-doped GaN film along with the charges that are formed in the direction perpendicular to the BSF plane. The bending of conduction band edge inside the BSF is due the electric field resulting from the polarization bound charge, whereas bands bend outside the BSF due to the accumulation and depletion of mobile charges. We approximate the accumulation charge as a sheet charge of density $n_{s}$ at the centroid $(t)$ of the charge distribution, i.e. $\rho_{\pi}(x)=e n_{s} \delta\left(x-x_{0}\right)$, where $x_{0}=d+t$, with $d$ being the width of a BSF and $e$ being the electron charge. If $x_{d}$ is the width of the depletion region, charge neutrality requires $n_{s}=x_{d} N_{d}$, where $N_{d}$ is the donor density. Energy conservation across the BSF leads to the relation

$$
\frac{e N_{d} x_{d}^{2}}{2 \epsilon_{s}}+\frac{e n_{s} t}{2 \epsilon_{s}}=\left(\frac{e \sigma_{\pi}}{2 \epsilon_{s}}-\frac{e n_{s}}{2 \epsilon_{s}}\right) d,
$$

where $\epsilon_{s}$ is the permittivity of the semiconductor. In the limit $x_{d} \gg(t, d)$, the depletion length is $x_{d} \simeq \sqrt{\sigma_{\pi} d / N_{d}}$. The depletion width $x_{d}$ increases with decreasing donor density and there exists a critical donor density $N_{d}^{\text {cr }}$ for which $x_{d}$ becomes equal to the distance between two stacking faults. As a result, the whole channel is depleted, and conduction ceases along the $x$-direction. The critical donor density above which the onset of conduction along $x$-direction occurs is given by $N_{d}^{\mathrm{cr}} \geqslant \sigma_{\pi} d n_{\mathrm{SF}}^{2}$. For a typical fault density $n_{\mathrm{SF}}=10^{5} / \mathrm{cm}$ and $d=0.8 \mathrm{~nm}$ for $\mathrm{GaN}$ [33], the critical doping density is $N_{d}^{\mathrm{cr}}=10^{16} / \mathrm{cm}^{3}$. 
(a)

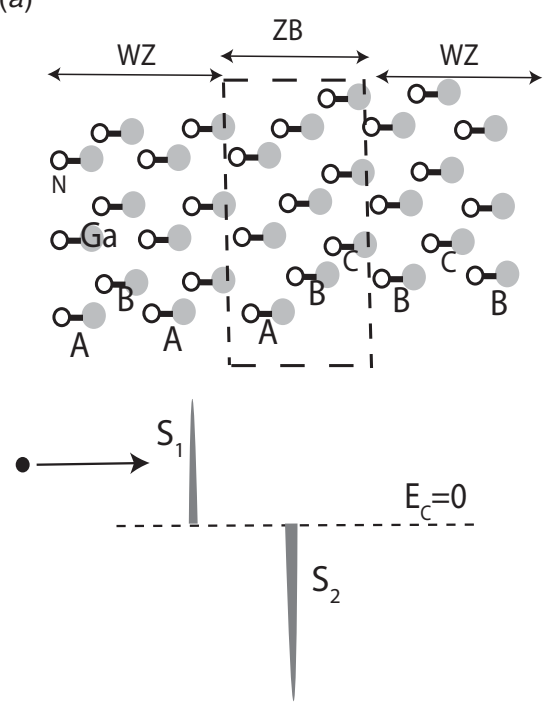

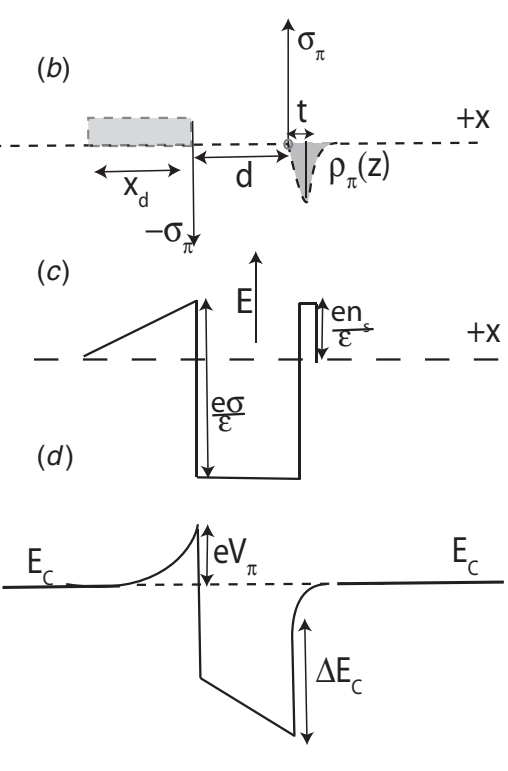

Figure 4. (a) Structure of BSFs, $(b)$ charges across the BSF, $(c)$ schematic diagram of electric field, $(d)$ conduction band diagram including band offset and $(e)$ delta-function model of barrier and QW. The figure is taken from [25].

3.1.2. Transport formalism through a BSF. In addition to the polarization charges at interfaces, the conduction band offset $\left(\Delta E_{c}\right)$ between $\mathrm{ZB}$ and $\mathrm{WZ}$ structures leads to $\mathrm{QW}$ in the fault region as shown in figure $4(b)$. For an applied bias, electrons tunnel through the barrier in the depletion region and the QW in the fault region, and then diffuse in the space between two consecutive stacking faults. To model the transmission coefficient of tunneling, we approximate the barrier and the QW as two delta functions as shown in figure 4(e) of strengths $S_{1}=e V_{\pi} x_{d}$ and $S_{2}=\left(\Delta E_{c}+e V_{\pi}\right) d$, where $V_{\pi}=\sigma_{\pi} d / 2 \epsilon_{s}$. The energy-dependent coefficient of transmission through a single delta function potential is an analytically solvable problem [34]. If $T_{\mathrm{tr}, 1}(\epsilon)$ and $T_{\mathrm{tr}, 2}(\epsilon)$ are the transmission coefficients for the barrier and the QW, respectively, then the total coefficient of incoherent transmission across the BSF is given by $T_{\mathrm{tr}}\left(\epsilon_{k}\right)=T_{\mathrm{tr}, 1}\left(\epsilon_{k}\right) T_{\mathrm{tr}, 2}\left(\epsilon_{k}\right)$ :

$$
T_{\text {tr }}\left(\epsilon_{k}\right)=\left[\frac{1}{1+\frac{m^{\star} S_{1}^{2}}{2 \hbar^{2} \epsilon_{k}}}\right] \times\left[\frac{1}{1+\frac{m^{\star} S_{2}^{2}}{2 \hbar^{2} \epsilon_{k}}}\right],
$$

where $\epsilon_{k}$ is the energy of the incoming electron and $m^{\star}$ is the effective mass at the band edge. In SF-free structures, the elemental current component along the $x$-direction for an electron with the velocity $v_{k}$ is given by $j_{x}^{k}=e v_{k}^{x} \delta f_{k}$, where $\delta f_{k}$ is the perturbation of the equilibrium Fermi-Dirac distribution caused by an applied electric field $F$. In the presence of $\mathrm{SFs}$, a fraction of these carriers given by equation (14) are transmitted through the barrier and the QW leading to an effective elemental current density $j_{k}^{\text {eff }}=T_{\text {tr }}\left(\epsilon_{k}\right) j_{k}^{x}$. Then, the total current density in the presence of SFs is given by

$$
J_{x}=2 e \int \frac{\mathrm{d}^{3} k}{(2 \pi)^{3}} T_{t r}\left(\epsilon_{k}\right) v_{k}^{x} \delta f_{k},
$$

where $v_{k}^{x}$ is the $x$ component of electron group velocity in the state $|\mathbf{r}, \mathbf{k}\rangle$ and the factor 2 takes spin degeneracy into account. For a small applied electric field along the $x$-direction, under the relaxation time approximation (RTA), $\delta f_{k} \approx\left(-\partial f_{0} / \partial \epsilon_{k}\right) \tau(k) v_{k}^{x} F$, where $f_{0}$ is the equilibrium Fermi-Dirac distribution function and $\tau(k)$ is the momentum relaxation time due to scattering from impurities, phonons, etc present in the material. Integrating equation (15) over the whole $k$-space and defining conductivity by the relation $J_{x}=\sigma(n, T) F$, one obtains the carrier concentration $(n)$ and temperature $(T)$-dependent conductivity in the presence of BSFs. The expression for conductivity across the BSF is then given by

$$
\begin{aligned}
& \sigma_{x x}(T, n)=\frac{3 n e^{2}}{8 \pi} \\
& \quad \times \frac{\int \mathrm{d} \epsilon_{k} \epsilon_{k}^{1 / 2} \cosh ^{-2}\left(\epsilon_{k} / 2 k_{B} T\right) T_{\mathrm{tr}}\left(\epsilon_{k}\right) \tau\left(\epsilon_{k}\right)\left(v_{x}^{k}\right)^{2}}{\int \mathrm{d} \epsilon_{k} \epsilon_{k}^{3 / 2} \cosh ^{-2}\left(\epsilon_{k} / 2 k_{B} T\right)},
\end{aligned}
$$

where $T$ is the equilibrium temperature, $k_{B}$ is the Boltzmann constant. Since the BSF does not break the periodic symmetry in the $y$-and $z$-directions, inserting $T_{\mathrm{tr}}\left(\epsilon_{k}\right)=1$ for all energies, a similar expression can be obtained for conductivity $\left(\sigma_{y y}\right)$ along the $y$-direction. For hole transport, $\Delta E_{c}$ should be replaced by the valence band offset $\left(\Delta E_{v}\right)$ in the strength of delta function potential $S_{2}$, and a hole effective mass should be used instead of the electron effective mass.

3.1.3. Scattering mechanisms. Equation (10) allows us to calculate experimentally measurable quantities such as conductivity and mobility in the presence of BSFs. As an application of the formalism constructed above, we investigate charge transport in p-type $m$-plane $(1 \overline{1} 100) \mathrm{GaN}$ in the presence of BSFs and compare the results with reported experimental data. Inclusion of different scattering mechanisms are necessary to evaluate the energy-dependent momentum relaxation time $\tau\left(\epsilon_{k}\right)$ appearing in equation (10). Due to the high activation energy of acceptors (Mg for p-type GaN has the activation energy $\sim 174 \mathrm{meV}$ [29]), a large fraction of the dopants remain neutral even at room temperature, acting as neutral impurity (NI) scatterers to hole transport. The fact 

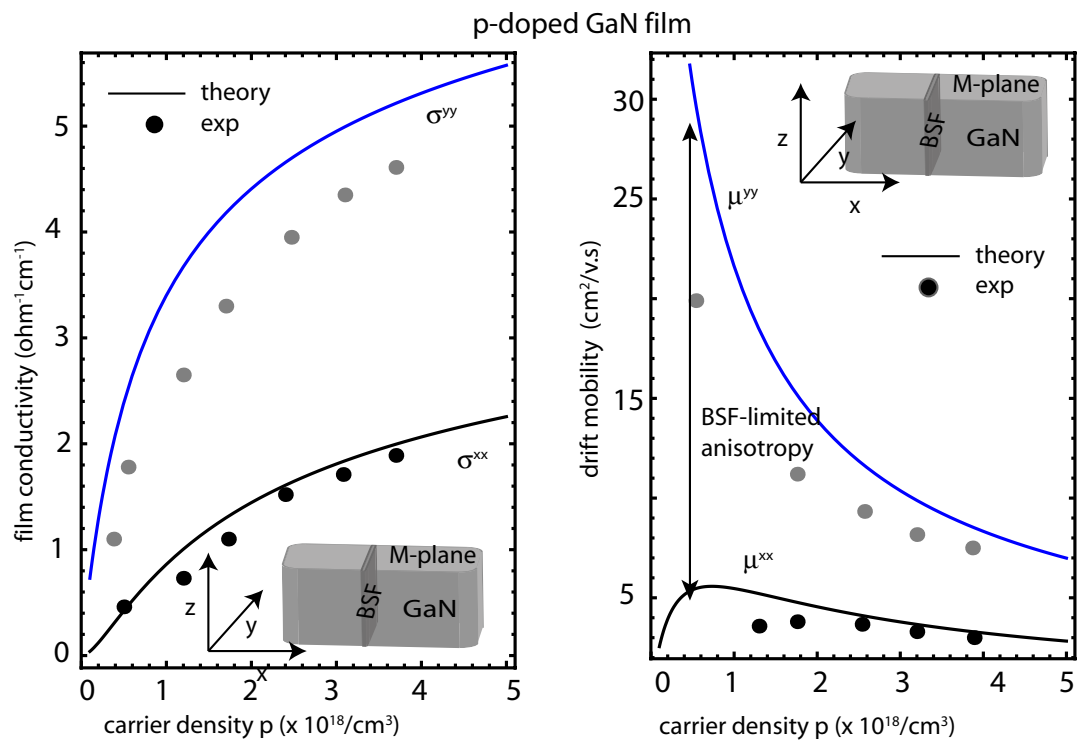

Figure 5. (a) Hole conductivity as a function of hole density: anisotropy is due to BSF, and (b) hole mobility as a function of hole density. Solid lines are theoretical values and solid circles are experimental values from [29]. Figures are taken from [25].

that a high doping density is required to achieve appreciable hole concentrations results in high density of NI making NIy scattering dominant even at room temperature. Accounting for the zero-order phase shift [35], the momentum relaxation time of NI scattering is $\tau_{\mathrm{NI}}^{-1}=20 N \hbar a_{0} / \mathrm{m}^{\star}$; where $N$ is the density of NI and $a_{0}=4 \pi \epsilon_{s} \hbar^{2} / m^{\star} e^{2}$ is the effective Bohr radius. Momentum relaxation time due to ionized impurity scattering $\left(\tau_{\text {imp }}\right)$ is calculated following the method of Brooks (BH) [36]. The fact that $\tau_{\text {imp }} \sim n_{\text {imp }}^{-1}$, where $n_{\text {imp }}$ is the density of ionized impurities, makes ionized impurity scattering important at high carrier concentrations due to the high density of ionized dopants. For electron-optical phonon momentum-relaxation time $\left(\tau_{\mathrm{ph}}\right)$, only phonon absorption has been considered due to the high optical phonon energy $\left(E_{\mathrm{op}}=0.092 \mathrm{eV} \gg k_{B} T\right)$ of $\mathrm{GaN}$. The resultant momentum-relaxation time is calculated using Mathiessen's rule, i.e. $\tau\left(\epsilon_{k}\right)^{-1}=\tau_{\mathrm{NI}}^{-1}+\tau_{\mathrm{imp}}^{-1}+\tau_{\mathrm{ph}}^{-1}$. For numerical calculations, we assume the valence band offset $\Delta E_{v}=0.06 \mathrm{meV}$ [32]. The hole effective mass of $\mathrm{GaN}$ is not well known and a wide range $m_{h}^{\star}=0.4-2.4 m_{0}$ is found in the existing literature [9]. Here, we have assumed the average hole effective mass $m_{h}^{\star} \sim 1.8 m_{0}$. The NI density is chosen to be $N=24 \times p$, where $p$ is free hole concentration in GaN. This choice is not ad hoc; measurements [29] show that the NI density $N=N_{A}-p \approx 20-25 \times p$ for a wide range of acceptor densities $N_{A}$.

3.1.4. Anisotropic hole transport. Figure 5(a) shows the calculated variation of $\sigma_{x x}$ (along the $c$-axis) and $\sigma_{y y}$ (parallel to $a$-axis) as a function of hole density at room temperature as solid lines juxtaposed with experimental [29] data shown as filled circles. It is apparent from the figure that the presence of BSF causes an appreciable reduction of $\sigma_{x x}$ compared to $\sigma_{y y}$, resulting in anisotropic hole transport. The corresponding hole mobility $\mu_{i i}=\sigma_{i i} / p e$, where $i=x, y$, is shown in figure $5(b)$ ). The anisotropy in hole mobility increases with decreasing hole density. This stems from the fact that in the limit $p \rightarrow 0$, the depletion width increases $\left(x_{d} \sim N_{A}^{-1 / 2}\right)$ and one approaches the critical acceptor density limit $N_{A}^{\text {cr }}$. As $x_{d}$ increases, the strength of the barrier $S_{1}$ increases, and $T_{\text {tr }}\left(\epsilon_{k}\right) \rightarrow 0$. This results in vanishing $\mu_{x x}\left(\sigma_{x x}\right.$; see figure 5).

\subsubsection{Anisotropic electron transport and the importance of} charged dislocation scattering. We next investigate the electron transport in n-doped samples in the presence of BSFs. Experiments [29] show that the electron mobility in the $m$-plane $\mathrm{GaN}$ decreases with decreasing electron concentration. This phenomenon is well described by charged dislocation scattering as pointed out by Weimann et al [37]. The large lattice mismatch between $\mathrm{GaN}$ and the substrate (for $\mathrm{GaN}$ on $\mathrm{SiC}$, lattice mismatch $2.9 \%$ ) leads to high dislocation densities. For the $m$-plane GaN grown on foreign substrates, TEM images reveal [38, 39] edge dislocation lines perpendicular to the $m$-plane (along the $\left[\begin{array}{llll}1 & 1 & 0 & 0\end{array}\right]$ direction)

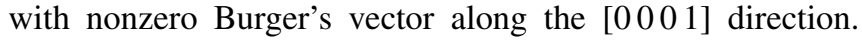
Each dislocation line acts as an acceptor-like trap with a linecharge density $\rho_{L}=e / a$, where $a$ is the $a$-lattice constant of $\mathrm{GaN}$. The electrons moving in the perpendicular plane to the dislocation line effectively feel a Coulomb potential $V(r)=$ $\left(\rho_{L} / 2 \pi \epsilon_{s}\right) K_{0}\left(r / L_{D}\right)$, where $K_{0}(\cdots)$ is the zeroth-order Bessel function of second kind and $L_{D}=\sqrt{k_{B} T \epsilon_{s} / e^{2} n^{\prime}}$ is the Debye screening length. Both free electrons and bound electrons are taken account in the effective screening concentration $\left(n^{\prime}\right)$ using the Brook's [40] formula $n^{\prime}=2 n-n^{2} / N_{D}$, where $n$ is the free carrier concentration. The momentum relaxation time $\left(\tau_{\text {dis }}\right)$ due to dislocation lines has been calculated by several authors [41, 37, 42] for three- and two-dimensional electron gases [43] in GaN. In the presence of BSFs, the $x$ component of conductivity at room temperature due to dislocation scattering can be calculated as

$$
\sigma_{x x}^{\mathrm{dis}}=\left(\frac{\epsilon_{s}}{e^{2}}\right)^{2} \frac{3 n e^{2} a^{2}\left(k_{B} T\right)^{3 / 2}}{4 \pi \sqrt{m^{\star}} n_{\mathrm{dis}} L_{D}} \mathcal{I}\left(n, \Delta E_{c}\right),
$$

where $n_{\text {dis }}$ is the dislocation density and $\mathcal{I}\left(n, \Delta E_{c}\right)$ is a dimensionless integral. The complex nature of the of the 

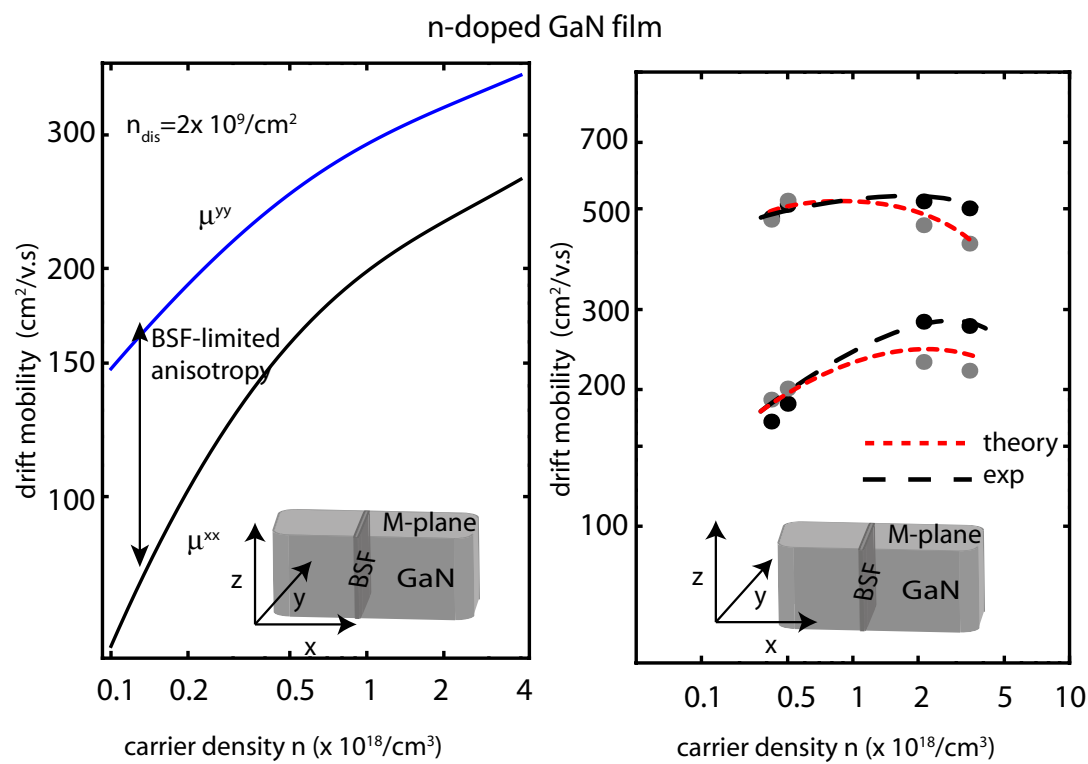

Figure 6. (a) Electron mobility as a function of electron density. (b) Comparison between theoretical values (dotted line) and experimental values (dashed line) of electron mobility at different values of electron concentrations. Figures are taken from [25].

integral $\mathcal{I}\left(n, \Delta E_{c}\right)$ prevents an analytical evaluation and we rely on a numerical evaluation. For $\Delta E_{c}=0.27 \mathrm{eV}$ [32] in the non-degenerate limit, $\mathcal{I}(n, 0.27) \simeq 6$, and $\mu_{x x} \sim \sqrt{n}$. Figure 6(a) shows the room temperature drift mobility with electron density for the dislocation density $n_{\mathrm{dis}}=2 \times 10^{9} / \mathrm{cm}^{2}$. It not only captures the mobility anisotropy due to BSF, but also the mobility variation with carrier concentration. The behavior is similar to those measured experimentally $[29,37]$. For calculations, we have used $m_{x x}^{\star}=m_{y y}^{\star}=0.22 m_{0}$, and a resultant momentum relaxation time $\tau^{-1}=\tau_{\mathrm{dis}}^{-1}+$ $\tau_{\mathrm{NI}}^{-1}+\tau_{\mathrm{imp}}^{-1}+\tau_{\mathrm{ph}}^{-1}$. Unlike p-doped GaN, the unintentionally doped $C$ atoms serve as NI with a measured density $N \sim$ $5 \times 10^{16} / \mathrm{cm}^{2}$ [29]. Measurements of [29] show that the free electron concentration measured using the Hall-effect techniques is lower than the actual dopant concentration in n-doped non-polar GaN samples. The difference between the dopant concentration and the free electron concentration is attributed to the acceptor traps associated with the dislocation lines. Moreover, the difference $\left(N_{D}-n\right) \sim n_{\text {dis }}$ varies from sample to sample indicating sample-dependent density of dislocations. Assuming a single trap per $a$-lattice constant, the volume density of trapped charge is $n_{\mathrm{dis}} / a$, and a charge balance equation can be written as

$$
n+\frac{n_{\text {dis }}}{a}=\frac{N_{D}}{1+\frac{n}{2 N_{C}} \exp \left(E_{D} / k_{B} T\right)},
$$

where $N_{C}=2\left(m^{\star} k_{B} T / 2 \pi \hbar^{2}\right)^{3 / 2}$ is the effective density of states and $E_{D}$ is the activation energy of the donor level. The screening effect on the donor activation energy is taken account by the empirical formula $E_{D}=E_{D 0}-\alpha N_{D}^{1 / 3}$ [44], where the screening parameter $\alpha=2.1 \times 10^{-5} \mathrm{meV} \mathrm{cm}$ and $E_{D 0}=28 \mathrm{meV}$ [44]. Using equation (12) and values of $N_{D}$ and $n$ from [29], the calculated dislocation densities are found to be in the range of $n_{\mathrm{dis}} \sim 1-10 \times 10^{8} \mathrm{~cm}^{-2}$. Electron mobility is determined using the calculated values of sample-dependent dislocation densities and compared to experimentally measured values as shown in figure $6(b)$ ). At low carrier densities, theoretical values are in agreement with experimental data, but they differ at high carrier concentrations.

3.1.6. Future scope. At low carrier densities, $x_{d}$ is large; the assumption of the delta function barrier breaks down. In such a carrier-density regime, one should solve the Schrödinger equation numerically to obtain exact transmission coefficient $T_{\text {tr } 1}(\epsilon)$. Moreover, at low doping levels (when $x_{d}$ is large) or for high stacking fault density, carriers are confined in a quasi-two-dimensional space rather than moving in three dimensions. Hence, one should use a two-dimensional analog of equation (10) for an exact evaluation of $\mu_{y y}$. In addition to that the localized states in BSFs can contribute in the transport. Recently, it was reported in [45] that for GaInN/GaN $m$-plane QWs, localization of carriers in BSFs shows quantumwire-like (QWR) features intersecting the QW. Carriers in the localized states contribute to the $\mu_{x x}$ by the thermionic process and to $\mu_{y y}$ by the diffusive process as shown in figure 7 .

3.1.7. Charge transport in bulk semi-polar nitrides. The transport formalism described above for GaN can be applied to all other nitride semiconductors in the presence of planar defects if the band offsets and the polarization differences between the WZ and the cubic phase are known. For semipolar bulk unstrained nitrides, the in-plane projection of the spontaneous polarization determines the bound charge density $\sigma_{\pi}$ at the interfaces of a BSF and alters the strength of the barrier $\left(S_{1}\right)$ and the QW $\left(S_{2}\right)$ created by a BSF. For example, if BSFs are oriented along the $\left(\begin{array}{llll}0 & 0 & 1 & 0\end{array}\right)$ direction, the in-plane polarization perpendicular to the BSF for a semi-polar plane at an angle $\theta$ with the $c$-axis is $\boldsymbol{P}=\boldsymbol{P}_{\mathrm{sp}}^{w} \sin \theta$, where $\boldsymbol{P}_{\mathrm{sp}}^{w}$ is the spontaneous polarization in the $\mathrm{WZ}$ structure parallel to the $c$-axis. Such calculations can shed light on transport in semi-polar $\mathrm{GaN}$ in the presence of stacking faults. 


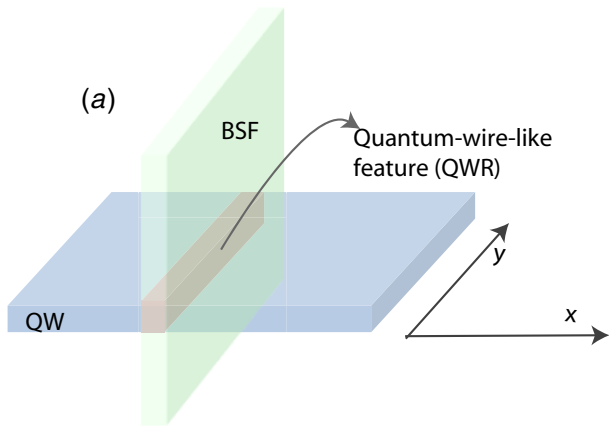

(b)

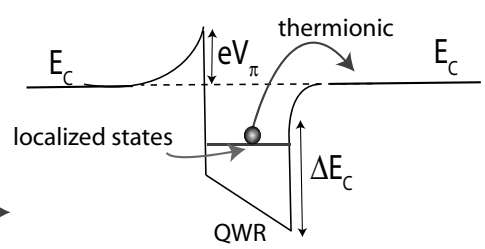

Figure 7. (a) QWR line structure in the presence of BSFs in nitride QWs and $(b)$ jump-over the barrier transport in the $x$-direction from the localized states in the BSF.

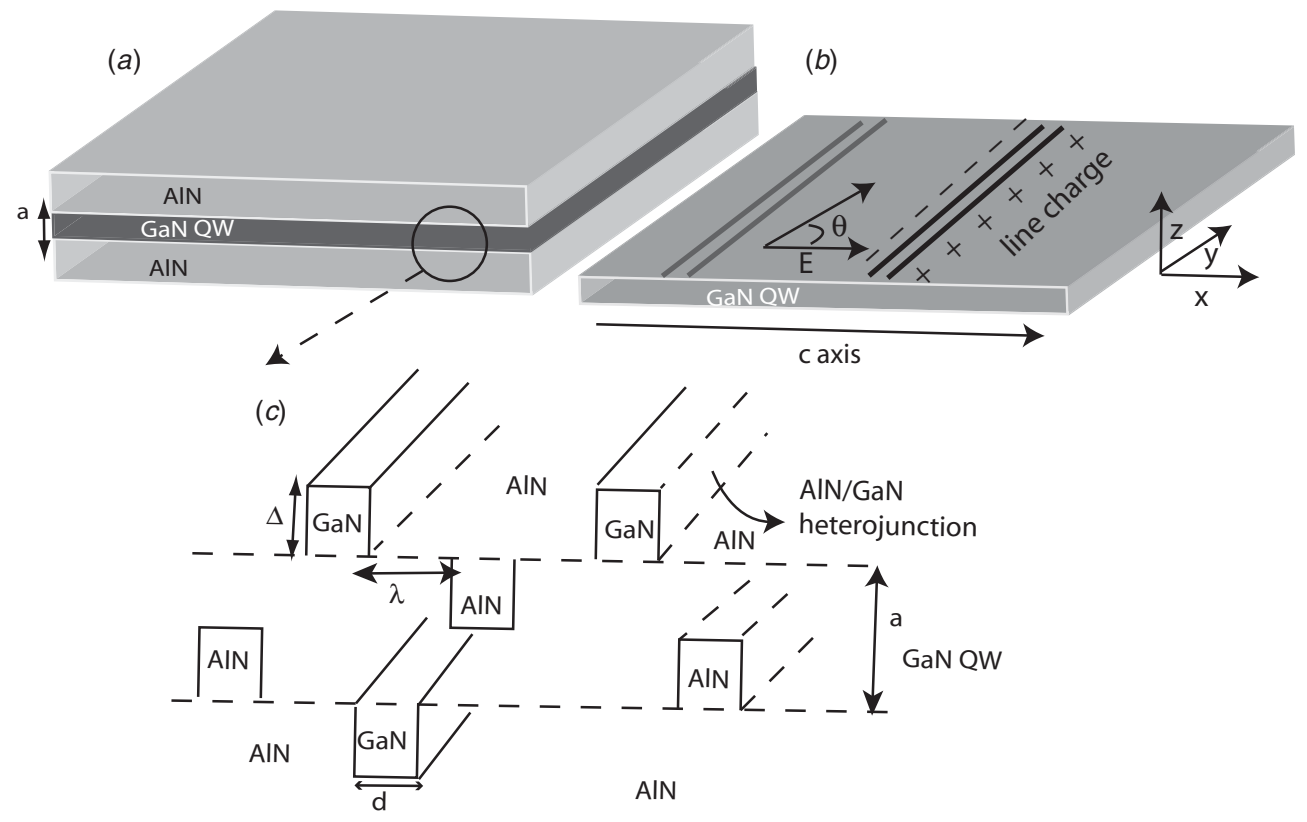

Figure 8. Non-polar GaN QW: (a) QW of width $a$ sandwiched between AlN barriers, (b) schematic diagram of IR at the GaN/AlN interface and $(c)$ polarization-induced line charge at each step edge of roughness. The figure is taken from [24].

\subsection{Charge transport in non-polar nitride heterostructures $Q W s$}

A characteristic feature of non-polar GaN surfaces grown directly on foreign substrates is extended stripe- or slatelike morphology perpendicular to the $c$-axis [26, 46-49]. The origins of this rough surface morphology have been attributed to (i) replication of substrate morphology [50], (ii) extended basal-plane stacking faults [26, 49], and (iii) anisotropic diffusion barrier of Ga adatoms [51, 52]. In the case of thick layers, the surface morphology does not affect the charge transport deep inside the bulk. But in the case of thin QWs, this rough surface morphology mingled with the in-plane polarization leads to anisotropic transport quantities [24], in addition to the anisotropy created by the stacking faults. In this section, we review the transport anisotropy originating by the rough surface morphology following the work reported in [24].

3.2.1. Interface roughness scattering from one-dimensional roughness. Let us consider a thin non-polar $\mathrm{GaN} \mathrm{QW}$ of thickness $a$ sandwiched between two aluminum nitride
(AlN) barrier layers as shown in figure $8(a)$. Note that the barrier layer could be any wider bandgap semiconductor. A common source of disorder for a non-polar GaN QW is striped surface morphology as mentioned above. We model this rough surface morphology as a local variation of QW thickness (see figure 8). The variation of QW thickness alone causes local shifts of the conduction band edge, resulting in carrier scattering commonly known as interface roughness (IR) scattering in the literature. The IR can be modeled by local thickness fluctuations $\Delta(x)$ of the non-polar GaN QW with a spatial correlation $\langle\Delta(x) \Delta(0)\rangle=\Delta^{2} \exp [-|x| / \sqrt{2} \Lambda]$ [53], where $\Delta$ is the average height of roughness and $\Lambda$ is the in-plane correlation length between two roughness steps. Denoting the envelope function of conduction electrons in the $n$th sub-band of GaN QWs as $|n, k\rangle=$ $\sqrt{2 / a} \sin (\pi n z / a) \mathrm{e}^{\mathrm{i} \vec{k} \cdot \vec{r}} / \sqrt{L_{x} L_{y}}$, the square of the unscreened intra-sub-band IR matrix element of scattering from the initial momentum state $\mathbf{k}_{\mathbf{i}}\left(k_{i}, \theta\right)$ to a final momentum state $\mathbf{k}_{\mathbf{f}}\left(k_{f}, \theta^{\prime}\right)$ in the $m$ th sub-band can be written as

$$
\left|v_{\mathrm{IR}}\left(q_{x}\right)\right|^{2}=\frac{m^{2}}{L_{x}}\left(\frac{\pi^{2} \hbar^{2} \Delta}{m^{\star} a^{3}}\right)^{2} \frac{2 \sqrt{2} \Lambda}{2+\left(q_{x} \Lambda\right)^{2}} \delta_{q_{y}, 0},
$$


where $\mathbf{q}=\mathbf{k}_{\mathbf{f}}-\mathbf{k}_{\mathbf{i}}, L_{x}$ and $L_{y}$ are the macroscopic lengths of the QW in $x$ - and $y$-directions, $\delta_{(\ldots)}$ is the Kronecker delta function, and $\hbar$ is the reduced Planck constant.

\subsubsection{Polarization induced line-charge scattering. The} thickness modulation of the QW leads to the appearance of GaN/AlN heterojunctions along the $Q W$ plane at each roughness center as depicted in figure $8(b)$. The difference of in-plane polarization of GaN and AlN creates line-charge dipoles by inducing fixed charges at opposite faces of each step as shown in figure $1(b)$. Assuming each rough step is infinitely extended along the $y$-direction (see [26, 46-49]), the electrostatic potential at any point $\mathbf{r}(x, y, z)$ in the QW arising from a polarization line-charge dipole of a roughness step at a point $\left(x_{i}, 0\right)$ of height $\Delta\left(x_{i}\right)$ and lateral width $d$ is given by [24]

$$
\begin{aligned}
V(x, y, z) & =v_{+}(x, y, z)-v_{-}(x, y, z) \\
& =\frac{e \lambda_{\pi}}{4 \pi \epsilon_{0} \kappa}\left[\ln \frac{\left(x-x_{i}-d / 2\right)^{2}+z^{2}}{\left(x-x_{i}+d / 2\right)^{2}+z^{2}}\right],
\end{aligned}
$$

where $\epsilon_{0}$ is the free-space permittivity, $\kappa$ is the relative dielectric constant of GaN and $\lambda_{\pi}\left(x_{i}\right)=\left|P_{\mathrm{GaN}}-P_{\mathrm{AlN}}\right| \Delta\left(x_{i}\right) / e$ is the effective line-charge density. Note that the potential is independent of $y$ due the symmetry of the problem. The scattering matrix element of transition from the state $\left|n, k_{i}\right\rangle$ to the state $\left|m, k_{f}\right\rangle$ can be written as $v\left(x_{i}, q\right)=$ $\left\langle m, k_{f}|v(x, y, z)| n, k_{i}\right\rangle$, where

$v\left(x_{i}, q\right)=\left(\frac{e \lambda_{\pi}}{\epsilon_{0} \kappa\left|q_{x} d\right|}\right) \mathrm{e}^{-\mathrm{i} q_{x} x} \sinh \left(\frac{\left|q_{x} d\right|}{2}\right) F_{n m}\left(q_{x} a\right) \times \delta_{q_{y}, 0}$.

Here, $F_{n m}\left(q_{x} a\right)$ [54] is the form factor arising from the quasi-2D nature of the electron gas. In our calculation of linecharge scattering, we neglect the effect of image charges at the dielectric interfaces as both $\mathrm{GaN}$ and AlN have similar dielectric constants $(\approx 9)$. Equation (15) represents the Fouriertransformed electrostatic potential from a line-charge dipole associated with a single roughness step. As two steps are correlated, the dipole potential arising from them are also correlated. If there are $N$ numbers of roughness steps, the square of the matrix element of the dipole potential summed over all scatterers is given by [24]

$$
\left|v_{\text {dip }}\left(q_{x}\right)\right|^{2}=v(0, q)^{2} L_{x} n_{\text {dip }}\left[1+\frac{2 \sqrt{2} n_{\text {dip }} \Lambda}{2+\left(q_{x} \Lambda\right)^{2}}\right],
$$

where $n_{\text {dip }}=N / L_{x}$ is the average roughness density $\left(\mathrm{cm}^{-1}\right)$ at the interface.

\subsubsection{Isotropic scattering mechanisms. Both IR and the line-} charge scattering mechanisms described above are anisotropic as the perturbation potentials break the planar symmetry. The other two important scattering mechanisms are (i) remote charge impurity (RI) scattering, and (ii) polar optical phonon (POP) scattering. We note that this is a non-polar QW, so unlike polar QWs, such conductive channels do not form due to the absence of polarization in the growth direction. To provide free carriers in the well, the $\mathrm{QW}$ is doped remotely. If $t_{0}$ is the distance between the QW and the remotely doped layer, the unscreened matrix element of scattering can be written as [55]

$$
\left|v_{\mathrm{RI}}(q)\right|^{2}=\left(\frac{e^{2} F_{m n}(q a)}{2 \epsilon_{0} \kappa}\right)^{2} \frac{\mathrm{e}^{-2 q t_{0}}}{q^{2}} .
$$

Optical phonon scattering in non-polar and semi-polar nitrides is similar to the phonon scattering in polar nitrides. POP scattering rates under the Davydov-Shmushkevich scheme $\left(\hbar \omega_{0} \gg k_{B} T\right)$, where phonon emission is assumed to be instantaneous, has been analytically calculated by Gelmont et al [56].

3.2.4. Transport quantities in the presence of anisotropic scatterers. To investigate the effect of anisotropic scattering events on experimentally measurable transport quantities (such as electron mobility, conductivity, etc), it is sufficient to consider carrier transport along the two principal directions $x$ and $y$. Transport coefficients in any arbitrary direction, in principle, can be obtained by a coordinate transformation. Transport quantities in the presence of anisotropic scattering are calculated following the formalism of Schliemann and Loss (SL) [57]. The carrier mobility in the SL scheme for a degenerate system is [24]

$$
\mu_{x x}=\frac{e}{\pi m_{x x}^{*}} \int_{0}^{2 \pi} \mathrm{d} \theta A^{\|}\left(k_{f}, \theta\right) \times \cos ^{2} \theta
$$

where $m_{x x}^{*}$ is the carrier effective mass in GaN along the $x$-axis and $A^{\|}$is given in [57]. A similar expression can be derived for $\mu_{y y}$.

The fact that anisotropic scatterers do not hinder carriers moving along the $y$-direction, in principle, should result in a higher mobility along the $y$-direction. Figure 9 captures this anisotropy. For numerical calculations, a nominal set of parameters are used to describe the $\mathrm{GaN} \mathrm{QW}$ roughness: $(\Delta, d)=(3.19,5.2) \AA$ and an isotropic effect mass of electron $m_{x x}^{*}=m_{y y}^{*}=0.2 m_{0}$ is assumed. With increasing Fermi energy both IR and line-charge scattering rates decrease, which in effect, reduces mobility anisotropy with the increasing carrier density $n_{s}$ as illustrated in figure 9(a). Similarly, for wide QWs, IR $\left(\mu_{\mathrm{IR}} \sim a^{6}\right)$ and line-charge scattering are unimportant and POP scattering is the dominant scattering mechanism. Consequently, $\mu_{x x}$ and $\mu_{y y}$ tend to approach the same limiting value determined by the POP scattering rate, and mobility anisotropy is completely washed out as seen in figure $4(b)$ for $a>7 \mathrm{~nm}$. This indicates that for bulk non-polar nitrides, transport anisotropy primarily originates from BSFs.

\subsubsection{Strain-induced piezoelectric polarization in semi-polar} and non-polar QWs. In the above analysis, strain was not taken into account. Semi-polar and non-polar nitride QWs grown on lattice mismatched substrates will have different line-charge density $\lambda_{\pi}$ at the roughness centers compared to the unstrained case. This is due to the straininduced piezoelectric polarization $\boldsymbol{P}_{\mathrm{pz}}$. The magnitude of this piezoelectric polarization varies with crystal planes due to the angular dependence of the strain components as mentioned in 

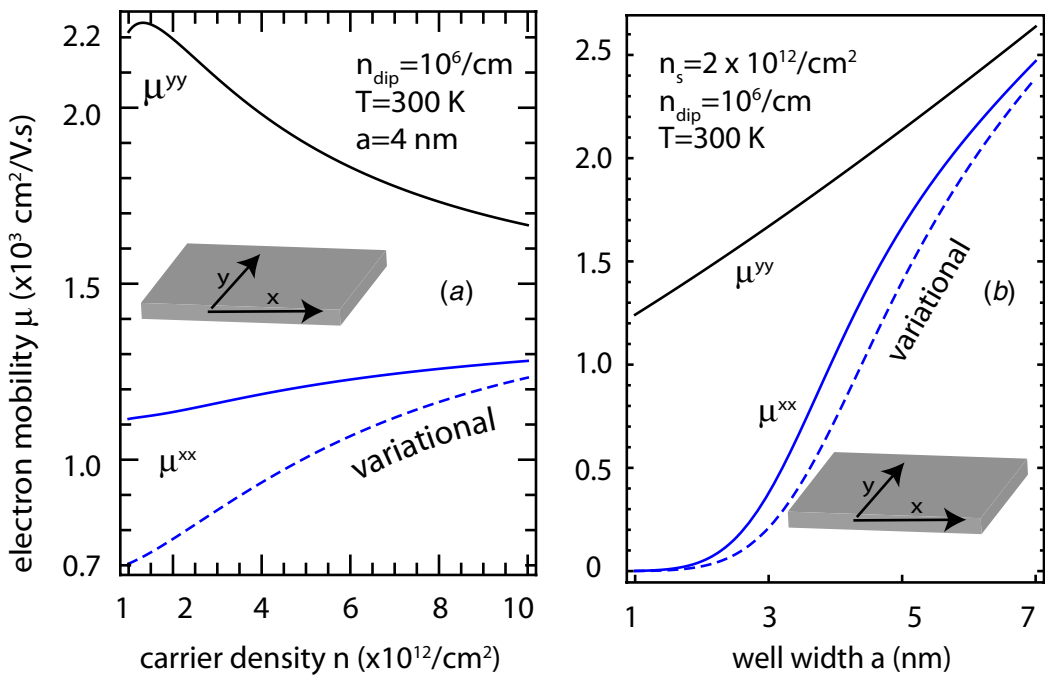

Figure 9. Electron mobility along two principal axes $x$ and $y:(a)$ as a function of carrier densities and $(b)$ as a function of well width. Note that the mobility anisotropy decreases with increasing carrier density or well width. Dashed blue curves represent longitudinal mobility calculated using the variational technique [58]. Figures are taken from [24].
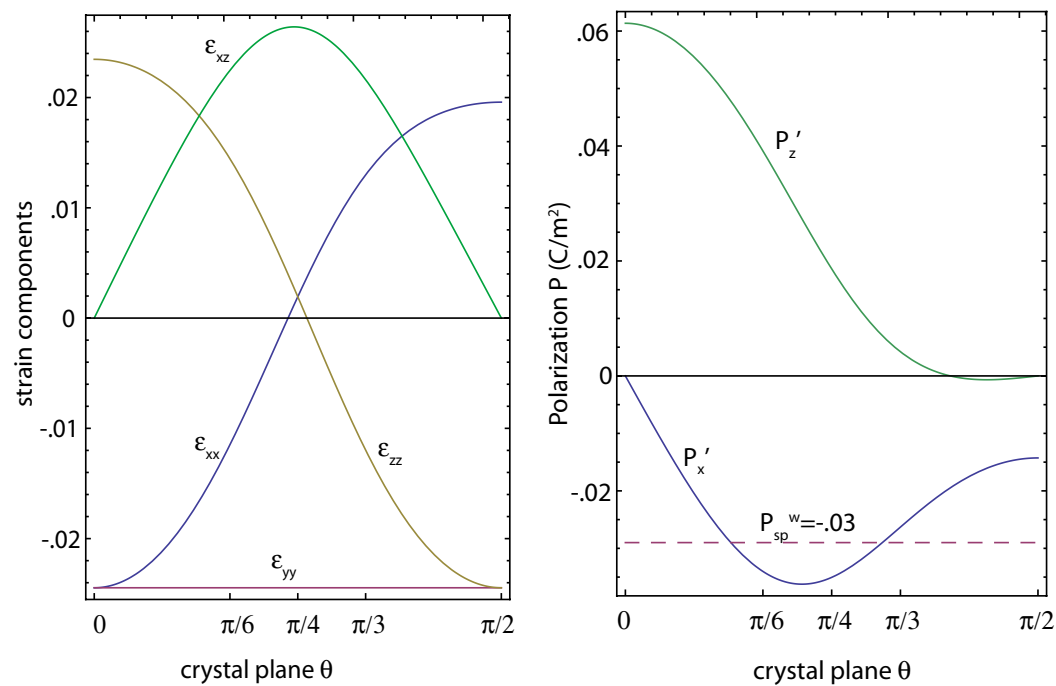

Figure 10. (a) Strain components with crystal orientation in the $(x, y, z)$ coordinate system and $(b)$ in-plane total polarization components for GaN on AlN.

section 2.1. For a general growth orientation, the polarization component in the $(x, y, z)$ coordinates are given by [20]

$$
\begin{aligned}
& P_{x}=2 e_{15} \epsilon_{x z}, \\
& P_{y}=0, \\
& P_{z}=P_{s p}^{w}+e_{31}\left(\epsilon_{x x}+\epsilon_{y y}\right)+e_{33} \epsilon_{z z},
\end{aligned}
$$

where $e_{i j}$ are the piezoelectric tensor elements, and the strain components $\epsilon_{i j}$ are given in section 2.1. The polarization component in the unprimed coordinates (the coordinate system associated with the growth plane) is now given by $\boldsymbol{P}^{\prime}=\mathcal{U}^{T} P$ and they are

$$
\begin{aligned}
& P_{x}^{\prime}=P_{x} \cos \theta-P_{z} \sin \theta, \\
& P_{y}^{\prime}=0, \\
& P_{z}^{\prime}=P_{x} \sin \theta+P_{z} \cos \theta .
\end{aligned}
$$

Figure 10 shows the different strain components and the in-plane polarization components for different crystal orientations of $\mathrm{GaN}$ on $\mathrm{AlN}$ substrate. Note that the presence of strain alters the in-plane polarization component $\left(P_{x}^{\prime}\right)$ responsible for line-charge scattering.

3.2.6. Transport in stacking fault-free semi-polar and nonpolar nitrides. Stacking fault-free high-quality semi-polar and non-polar nitrides and related compounds are currently under development [59-61]. It is apparent that with decreasing BSF density, transport anisotropy will decrease and eventually vanishes for BSF-free non-polar and semi-polar GaN films. Charge transport in BSF-free bulk nitrides is diffusive and the transport quantities are determined by appropriate scattering mechanisms described in section 3.1.3.

In BSF-free semi-polar and non-polar QWs, it is more likely to assume localized surface roughness (LSR) randomly distributed at the QW/barrier interface as shown in figure 11(a). The heterojunctions associated with LSRs lead to tiny in-plane 


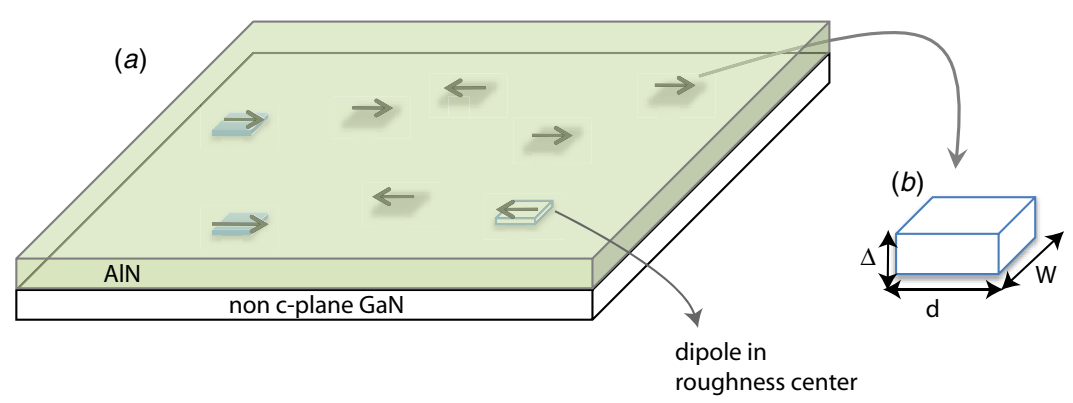

Figure 11. IR and in-plane dipoles in BSF-free non-polar and semi-polar nitride QW.
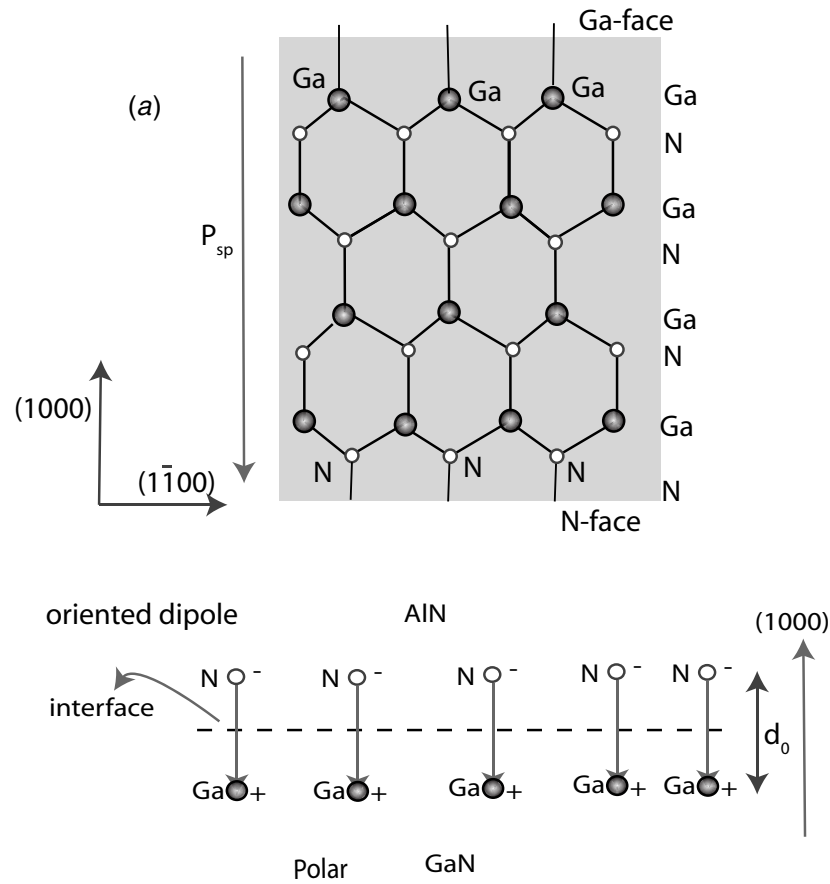

(b)
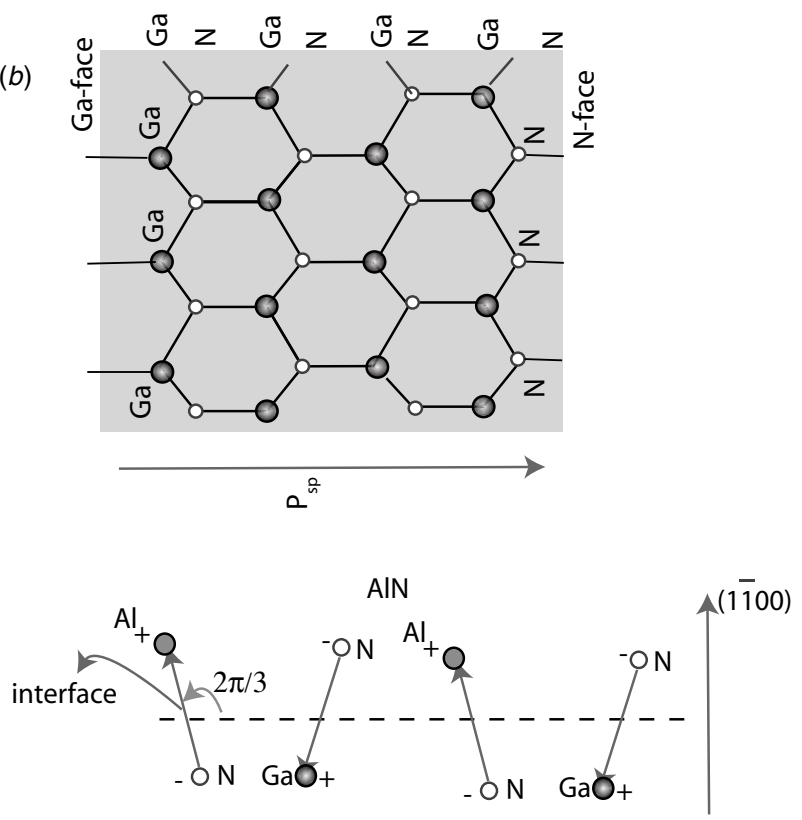

Non-Polar GaN

Figure 12. Interface dipoles at the AlN/GaN interface for $(a)$ polar growth and $(b)$ non-polar growth. Note that for the polar growth, the dipoles are perfectly oriented.

dipole moments denoted by tiny arrows in the figure randomly positioned at the interface. The magnitude of the tiny dipole moment is $P=\left|P_{\mathrm{QW}}-P_{B}\right| d w \Delta$, where $P_{\mathrm{QW}(B)}$ is the in-plane polarization of the QW (barrier) material, $d, w$ and $\Delta$ are the average length, the width and the height of a roughness as shown in figure $11(b)$. The potential created by these dipoles act as an extra source of the isotropic scattering mechanism in BSF-free non-polar and semi-polar nitride QWs.

\subsection{Charge-transfer interface dipole moments in polar, semi-polar and non-polar III-V nitride transistors}

An important difference between the polar and non-polar (or semi-polar) nitride heterojunctions is the interface dipole scattering associated with the interface bonds of the heterojunction. The origin of the interface dipole is due to the charge sharing between the atoms of dissimilar electronegativity upon the formation of interface bonds originally pointed out for the $\mathrm{Si}-\mathrm{SiO}_{2}$ interface [62]. Figure 12 shows the interface of $\mathrm{AlN} / \mathrm{GaN}$ heterojunction along with the interface dipoles for polar and non-polar growth. For polar $\mathrm{GaN}$, the interface atoms are either $\mathrm{Ga}$ (for Ga-face growth) or $\mathrm{N}$ (for $\mathrm{N}$-face growth). As a result, the interface dipoles are perfectly oriented as shown in figure $12(a)$. This leads to shift in the threshold voltage in transistors $\Delta V_{\text {th }}=e n_{d} d_{0} /\left(\epsilon_{0} \kappa\right)$, where $n_{d}$ is the dipole density (per unit area) and $d_{0}$ is the interface bond length. For typical values of $n_{d} \approx 10^{14} / \mathrm{cm}^{2}$ and $d_{0} \sim \AA$, interface charges lead to a non-negligible threshold voltage shift $\Delta V_{\text {th }}=0.3 \mathrm{~V}$. For a perfect interface, these interface dipoles do not scatter carriers as they have the same periodicity as the crystal. But if the periodicity is broken by vacancies, defects, or broken bonds, transport quantities are affected by scattering from this interface dipoles.

For semi-polar and non-polar growth, the interface of $\mathrm{GaN}$ has both $\mathrm{Ga}$ and $\mathrm{N}$ atoms that alternately appear at the interface plane as shown in figure $12(b)$. As a result, interface dipole moments are oriented randomly and their effects average out to zero. The detailed mathematical description of the effect of the interface dipole scattering for polar and non-polar growth remains an open problem. 

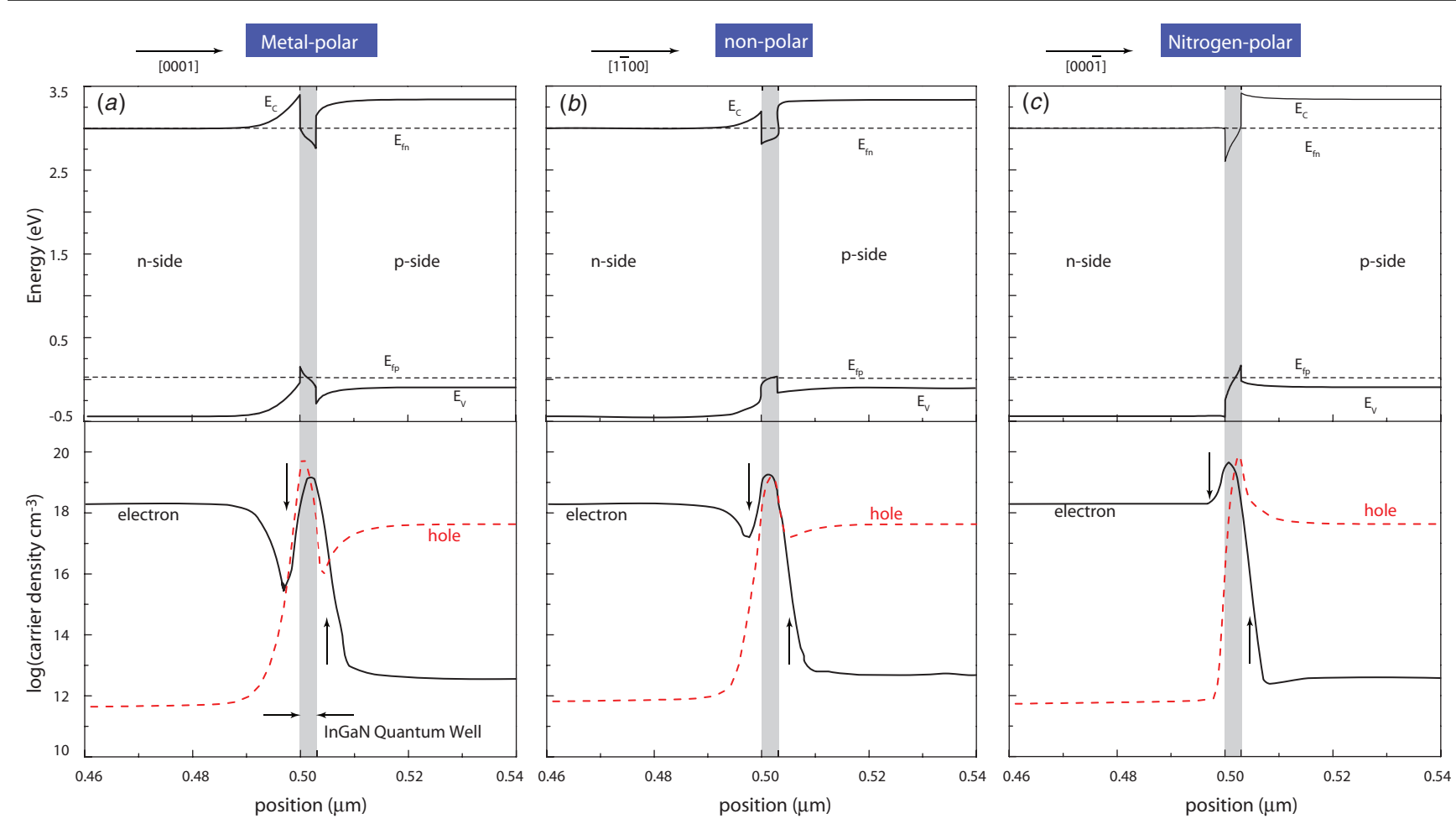

Figure 13. Energy band diagrams of $(a)$ metal-polar, $(b)$ non-polar and $(c) \mathrm{N}$-polar $\mathrm{n}$-GaN/InGaN/p-GaN QW LED structure. The energy band diagrams are calculated self-consistently at a forward bias voltage of $3 \mathrm{~V}$. The purpose of the calculation is to illustrate the effect of polarity and crystal orientation on carrier injection and transport in such devices. Typical LEDs incorporate multiple QWs and electron blocking layers, which are not shown for the sake of clarity.

\section{Polarity-dependent carrier transport effects in QW LEDs}

Semi-polar and non-polar crystal orientations are attractive for III-Nitride optical devices. Heterostructure QWs in such orientations exhibit lower built-in polarization fields than the polar counterparts. This potentially increases the electron/hole wavefunction overlap and boosts the oscillator strength of the interband transition. A high oscillator strength boosts the internal quantum efficiency of the spontaneous radiative recombination $\eta_{r}$ by increasing radiative recombination according to the relation $\eta_{r}=\tau_{r}^{-1} /\left(\tau_{r}^{-1}+\tau_{n r}^{-1}\right)$.

The total efficiency $\eta_{\text {tot }}$ of a light emitting device also depends on the efficiency of carrier injection $\eta_{\text {inj }}$ and the photon extraction efficiency $\eta_{\text {extr }}$, i.e. $\eta_{\text {tot }}=\eta_{\text {inj }} \eta_{r} \eta_{\text {extr }}$. The injection efficiency depends on the nature of injection of electrons and holes into the active region $(\mathrm{QW})$. This process of carrier injection and retention in the QW depends on the crystal orientation of the QWs due to the polarization sheet charges and the resulting electric fields. We include a brief discussion of the transport effects on the injection efficiency and its dependence on various possible crystal orientations. To put things in perspective, we have self-consistently simulated a simple single $\mathrm{QW}$ InGaN sandwiched in a $\mathrm{GaN}$ p-n junction. The QW is $3 \mathrm{~nm}$ thick and is composed of $\mathrm{In}_{0.15} \mathrm{Ga}_{0.85} \mathrm{~N}$. The simulation is performed by self-consistently solving the Poisson and Schrödinger equation with the charge-transport equation at a forward bias, using the commercially available design tool Crosslight [63]. Three orientations of the crystal were chosen to cover the entire range of polarization fields possible.

Figure 13 shows the energy-band diagrams of the prototype LED structure under a forward bias of $V_{F}=3 \mathrm{~V}$, which is the splitting of the electron and hole quasi Fermi-levels $\left(E_{f n}-E_{f p}=q V_{F}\right)$. Before we proceed with a discussion of the differences between the three orientations, we note that a metal-polar structure would nominally be identical in every respect to the nitrogen-polar structure if there was no doping, since that would be equivalent to flipping the crystal around, which surely cannot change the physics inside. However, LEDs typically have the more resistive ptype-doped layer on the top, which is the right side of the energy-band diagrams in figure 13. This constraint gives different properties of the metal-polar structure from the Nitrogen-polar structure. Figures 13(a)-(c) show the energy band diagrams and the electron and hole densities for LED structures oriented along the metal-polar, non-polar, and Npolar orientations, respectively. The corresponding cases for semi-polar orientations are intermediate between these cases.

We first note that for the metal-polar case, transport of electrons into the QW from the n-type injector layer meets a potential barrier that is larger than the non-polar structure due to a negative polarization sheet charge at the QW/n-type interface. This barrier is nearly eliminated for the $\mathrm{N}$-polar structure since the positive polarization sheet charge lowers the barrier for electron injection. Thus, carrier injection into the QW is most efficient for the N-polar structure. However, the retention of electrons in the QW also suffers in the N-polar structure due to the elimination of the barrier; 
electrons can leak back into the n-injector region, causing weaker confinement. Carriers outside the active regions lead to loss, since even if they lead to the interband radiative recombination, the photons emitted are not at the desired wavelength. Similarly, electron-wavefunction 'leakage' into the p-layers is successively lowered as one moves from metalpolar through non-polar to $\mathrm{N}$-polar structures. The arrows point to the effect of the net steady-state carrier densities for the three structures. Exactly same arguments apply to holes.

For the carriers that are retained, the electron-hole overlap in the active region is the best for the non-polar structure due to the absence of the polarization field. The cases of metal versus $\mathrm{N}$-polar QWs are worth further discussion under high current injection (which is associated with 'efficiency droop' observed in LEDs). For both the metal-polar and N-polar structures, the dipole formed by the electrons and holes in the QW is opposite in direction to the polarization charge sheet dipole at the QW/clad layer interfaces. For the non-polar structure, there is almost no such dipole. As the current injection is increased, the carrier dipoles increase in strength. For both polar QWs, the injected dipole tries to screen the polarization dipole, which will lead to a better overlap, low spectral shift and, thus, more efficient radiative recombination. However, the carrier leakage or 'overshoot' across the QW will reduce for the metal-polar structure as current injection increases, whereas it will get worse for the N-polar structure compared to low current injection situations. When compared against each other, N-polar structures have low leakage across, and worse leakage to the same side of the QW than metal-polar QW LEDs. In general, from the qualitative arguments, it appears that the non-polar structure (or low-polarization semi-polar planes) offer the best compromises over the polar variants. The metal-polar and N-polar structures have their relative merits and demerits. These arguments hold for emitters in the visible wavelengths.

As one moves to higher bandgaps for UV and deepUV optical emitters, the situation changes due the problem in doping. Due to difficulty in p-type (and to an extent also n-type) doping of high Al-containing AlGaN layers, the carrier-injection problem dominates the performance of the device. The N-polar orientation can help improving the carrier-injection process by using the polarization charges to effectively 'pull' in carriers into the active regions. In addition, one can use polarization-induced doping techniques in graded AlGaN structures for further boosting the performance [64-66].

\section{High-field transport in semi-polar and non-polar GaN}

At high electric fields, electrons gain enough kinetic energy to populate high-energy states of the energy bandstructure. Thus, crystal orientation effects play a major role in highfield or hot-electron transport properties. Such high fields occur in HEMTs in the channel near the drain side of the gate at high drain voltages. In optoelectronic devices designed for photon emission (LEDs, lasers), the fields are typically small in forward bias regimes and are screened by injected carriers. However, for photon absorption, devices are typically reverse biased, and high electric fields are necessary to sweep photogenerated carriers out of the absorbing layers before they recombine. This phenomenon occurs in UV photodetectors or in avalanche photodiodes (APDs). It is necessary to understand carrier transport properties along various orientations of the GaN crystal for such devices. To do so, an accurate energy bandstructure of $\mathrm{GaN}$ is essential.

Figure 14 shows the calculated energy bandstructure and various associated physical quantities along different crystal orientations of GaN. The bandstructures are provided by Professor C Van de Walle, the University of California, Santa Barbara, CA. The bandstructure is calculated using hybridfunctional DFT that is able to capture the correct bandgaps and has recently been used to explain various peculiarities of $\mathrm{GaN}$ devices, such as efficiency droop, among others. Of primary interest for high-field transport properties are three orientations: from $\Gamma \rightarrow A$, which is along the $\left(\begin{array}{llll}0 & 0 & 0 & 1\end{array}\right)$ polar orientation of the GaN crystal, the $\Gamma \rightarrow M$, which is along

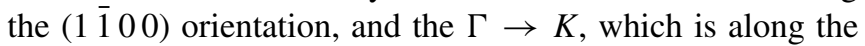
(11 $1 \overline{2} 0)$ orientation. The $\Gamma \rightarrow A$ direction is polar, whereas the $\Gamma \rightarrow M$ and the $\Gamma \rightarrow K$ orientation are non-polar. The bandstructure along the semi-polar direction (e.g. along $11 \overline{2} 2$ ) is not shown, but arguments similar to the discussion here should apply, with an intermediate polarization-induced field and charges at heterojunctions. To connect to devices, we note that vertical transport in $c$-plane metal-polar as well as $\mathrm{N}$-polar optical devices occurs in the $\Gamma \rightarrow A$ direction near the active regions. In access regions from side-contacts (e.g. in etched lateral n-type contacts), the transport is primarily in the non-polar $\Gamma \rightarrow M$ and $\Gamma \rightarrow K$ directions. On the other hand, for polar III-nitride HEMTs, the crucial part of transport is primarily in the non-polar $\Gamma \rightarrow M$ and $\Gamma \rightarrow K$ directions in the two-dimensional electron gas channels confined at the $\mathrm{Al}(\mathrm{InGa}) \mathrm{N} / \mathrm{GaN}$ heterojunctions.

Figures $14(c)$ and $(d)$ show the group velocities $v_{g}=$ $\hbar^{-1} \nabla_{k} E(k)$ for the electron and the heavy hole bands. The electron group velocity along the $\Gamma \rightarrow A$ and the $\Gamma \rightarrow M$ orientations look similar, reaching the maxima of $v_{g}^{\max } \sim$ $8 \times 10^{7} \mathrm{~cm} \mathrm{~s}^{-1}$ at wave vectors $k_{0} \sim 0.3 \times 10^{10} \mathrm{~m}^{-1}$. These inflection points in the bandstructure are indicated by open circles in figure 14(e). They occur at $\sim 1.1 \mathrm{eV}$ above the conduction band edge in the $\Gamma \rightarrow A$ direction, and at $\sim 1.5 \mathrm{eV}$ above the conduction band edge in the $\Gamma \rightarrow M$ direction. At the inflection point, the effective mass of carriers diverges, and at higher energies, it becomes negative. We note that the negative effective mass regions exist for the conduction band in the $\Gamma \rightarrow A$ and $\Gamma \rightarrow M$ directions, but not along the $\Gamma \rightarrow K$ direction. The electron group velocity along the $\Gamma \rightarrow K$ direction is also lower than the other orientations at high energies. For the valence band shown, there is an inflection point in the $\Gamma \rightarrow M$ direction.

Most of these high-energy states are not accessed in normal device structures under low bias regimes. This is especially true in GaN due to the very strong electronPOP interaction in the semiconductor. Owing to the light atomic mass of nitrogen, the optical phonon energy is high ( $\hbar \omega_{\mathrm{op}} \sim 92 \mathrm{meV}$ ), and owing to the high electronegativity of 

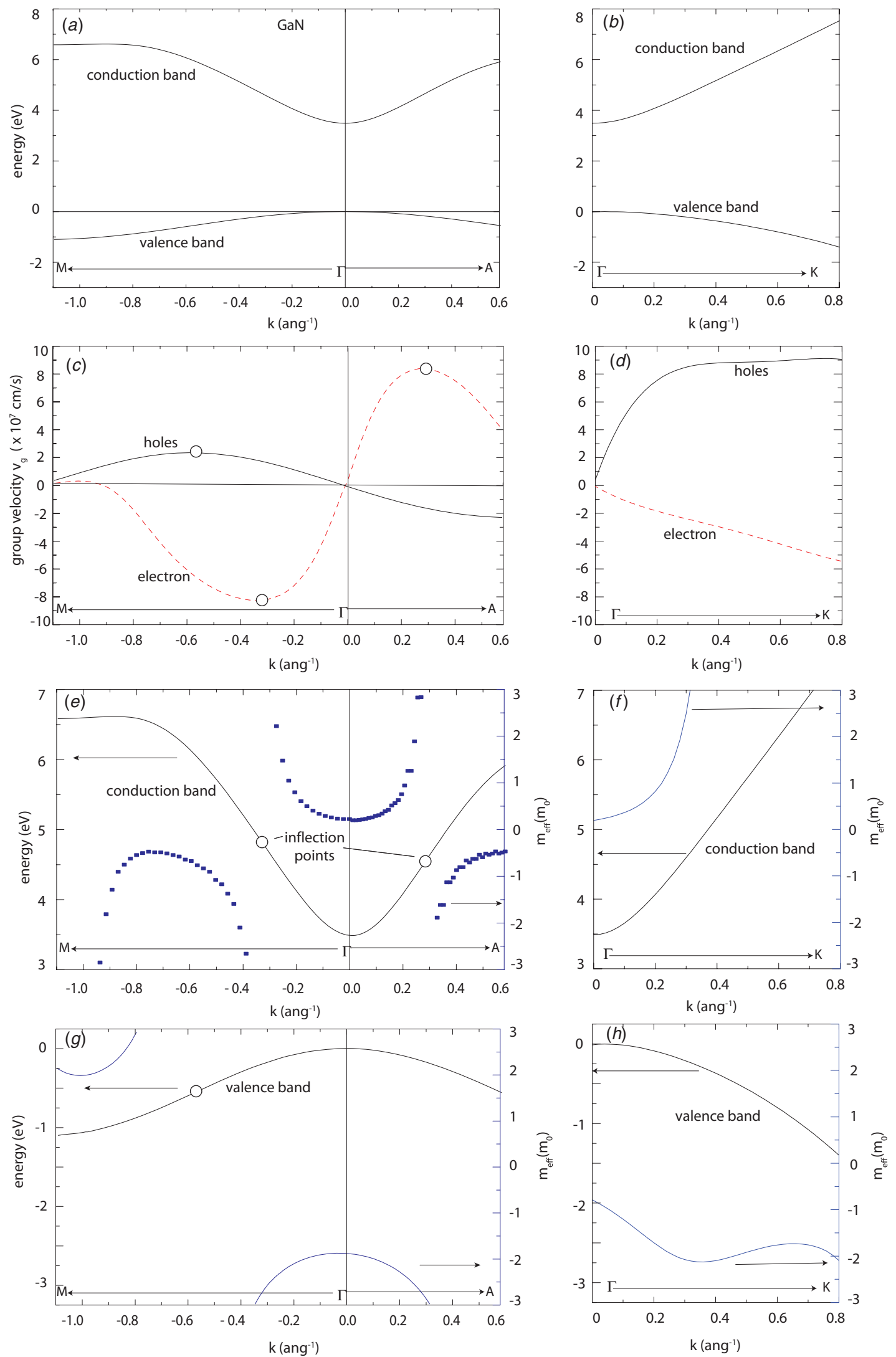

Figure 14. DFT-based energy bandstructure, group velocities and effective masses of the conduction and the heavy hole bands along various orientations of the GaN crystal. The inflection points in the bandstructure are also indicated by circles. 
nitrogen, the dipole moment of the $\mathrm{Ga}-\mathrm{N}$ bond is larger than other semiconductors. This leads to efficient optical phonon emission process at high fields, which prevents electrons from gaining high kinetic energies. Thus, if carriers are accelerated from the $\Gamma$ point at the bottom of the conduction band, for normal electric fields, they rarely get into the negative effective mass regions since fast phonon emission scatters them back to regions near the $\Gamma$ point. The group velocity of electron states with kinetic energy equal to one optical phonon energy is given by $v_{\text {op }} \sim \sqrt{2 \hbar \omega_{\mathrm{op}} / m^{\star}} \sim 4 \times 10^{7} \mathrm{~cm} \mathrm{~s}^{-1}$, and the thermal velocity is $v_{\text {th }} \sim \sqrt{k T / m^{\star}} \sim 1.5 \times 10^{7} \mathrm{~cm} \mathrm{~s}^{-1}$. The net ensemble velocities of carriers are expected to be around the thermal velocity, typically much lower than $v_{\text {op }}$. Since the bandstructure is mostly isotropic near the $\Gamma$ point, the velocities are not expected to depend significantly on the polarization direction in bulk materials.

This simplistic single-particle picture needs to be modified in the presence of a distribution of carriers. For example, in a HEMT with a high 2DEG density, the high degeneracy implies that mobile carriers do not start from the $\Gamma$ point energy which is the conduction band edge $E_{c}$. Instead, they start at energies $E_{F}=E_{\Gamma}+\pi \hbar^{2} n_{s} / m^{\star}$, which describes a Fermi circle in the $k$-space for the first energy sub-band. Here, $n_{s}$ is the 2DEG sheet density for the first sub-band; for high densities, the carrier degeneracy populates states at energies $0.1 \mathrm{eV}$ above the $\Gamma$ point at $n_{s} \sim 10^{13} \mathrm{~cm}^{-2}$ and $\sim 0.5 \mathrm{eV}$ for $n_{s} \sim 5 \times$ $10^{13} \mathrm{~cm}^{-2}$. At very large fields, it is likely that a fraction of carriers cross the inflection point. However, the fraction is expected to be small given the high phonon emission rate. On the other hand, for carrier transport across a sharp heterojunction, the situation can be quite different.

Consider a case where electrons are injected from the conduction band of AlGaN into GaN across an AlGaN/GaN heterojunction. The conduction band offset $\Delta E_{c}$ then acts as an energy cutoff leading to possible carrier injection directly into regions of the bandstructure very close to, or directly into the negative effective mass region. This could be achieved in a vertical heterostructure along the $\Gamma-A$ orientation, the typical direction of charge transport in optical devices. The effective mass mismatch will cause reflections, but the portion of carriers that get injected into the negative effective mass region can lead to current instabilities and corresponding current oscillations in the time domain. This process can be a recipe for generating ac power at very high frequencies. Such negative mass oscillations will compete against intervalley scattering, but from the bandstructure in figure 14, there exists a substantial energy separation between the inflection point and the next satellite valley in the $\Gamma \rightarrow A$ and the $\Gamma \rightarrow M$ directions. Thus, negative effective mass oscillators, which were initially proposed by Kroemer [67] and are yet to be realized, could possibly be achieved in GaN [68]. Such oscillations in principle can operate at higher frequencies than Gunn diodes since they are not limited by intervalley scattering rates.

We conclude the section on high-field transport with a brief description of tunneling in III-nitrides and the effect of polarization. For intraband tunneling transport such as in resonant tunneling diodes, or in superlattices in the form of minibands, non-polar directions are preferred [69]. Polarization causes a narrowing of the bandwidth of a superlattice miniband, implying a larger effective mass and thus less-efficient transport.

On the other hand, interband Zener tunneling is weak for bulk GaN due to the wide bandgap. Indeed, the high breakdown properties that enable high-voltage and highpower applications in electronics depend on the weakness of interband tunneling in bulk GaN. The dependence of interband Zener tunneling on the polarization orientation of the crystal is linked to the asymmetry of the valence bands, which are the source of electrons for such transport. This topic has not been studied extensively. Zener tunneling depends strongly on the driving electric field. Since polarization fields can be much higher than those achievable by traditional doping techniques, polarization-induced Zener tunnel junctions are possible along the most polar crystal orientations. Such tunnel junctions have been demonstrated recently and might have useful applications in the near future [70-72]. By the same token, interband tunneling is expected to be rather weak in similar heterostructures in the semi- and non-polar orientations. The variety of choices results in a rich range of tools that can be used as device building blocks based on the polarity and orientation of $\mathrm{GaN}$ crystals.

\section{Conclusions}

To conclude, this paper summarizes a number of transport phenomena that depend on the crystal orientation in polar semiconductors. The theory and models are expected to find usage not just for designing and understanding optical and electronic devices using III-V nitride semiconductors, but other polar heterostructures as well. One emerging material family is that of highly polar oxide crystals that exhibit polarization effects at heterojunctions [73]. As experimental work evolves with improvement and expansion of the availability of III-nitride crystals of various crystal orientations, corresponding new phenomena in chargetransport and optical properties are expected to emerge. The methods presented in this paper can be used as guidelines for understanding transport-related phenomena in such materials.

\section{References}

[1] Waltereit P et al 2000 Nature 406865

[2] Eastman L F and Mishra U K 2002 IEEE Spectrum 3928

[3] Khan M A, Bhattarai A, Kuznia J N and Olson D T 1993 Appl. Phys. Lett. 631214

[4] Nakamura S, Senoh M, Nagahama S, Iwasa N, Matsushita T and Mukai T 2000 Appl. Phys. Lett. 7622

[5] Wood C and Jena D 2008 Polarization Effects in Semiconductors: From Ab Initio Theory to Device Applications (New York: Springer)

[6] Jin Seo Im, Kollmer H, Off J, Sohmer A, Scholz F and Hangleiter A 1998 Phys. Rev. B 57 R9435

[7] Cai Y et al 2005 IEEE Electron Device Lett. 26435

[8] Chakraborty A, Baker T J, Haskell B A, Wu F, Speck J S, Denbaars S P, Nakamura S and Mishra U K 2005 Japan. J. Appl. Phys. 44 L945

[9] Piprek J 2007 Nitride Semiconductor Devices: Principles and Simulations (Weinheim: Wiley-VCH) 
[10] Vanfleet R, Simmons J, Maruska H, Hill D, Chou M and Chai B 2003 Appl. Phys. Lett. 831139

[11] Gardner N, Kim J, Wierer J, Shen Y and Krames M 2005 Appl. Phys. Lett. 86111101

[12] Haskell B A, Baker T J, McLaurin M, Wu F, Fini P T, Denbaars S P, Speck J S and Nakamura S 2005 Appl. Phys. Lett. 86111917

[13] Ng H 2002 Appl. Phys. Lett. 804369

[14] Baker T J, Haskell B A, Wu F, Fini P T, Speck J S and Nakamura S 2005 Japan. J. Appl. Phys 44 L920

[15] Takeuchi T, Amano H and Akasaki I 2000 Japan. J. Appl. Phys. 39413

[16] Ghosh S, Waltereit P, Brandt O, Grahn H T and Ploog K H 2002 Phys. Rev. B 65075202

[17] Kane E O 1957 J. Phys. Chem. Solids 1249

[18] Chuang S L and Chuang C S 1996 Phys. Rev. B 542491

[19] Lowdin P 1951 J. Phys. Chem. 191396

[20] Park S H and Chuang S L 1999 Phys. Rev. B 594725

[21] Park S H, Ahn D and Chuang S L 2007 IEEE J. Quantum Electron. 431175

[22] Scheibenzuber W G, Schwarz U T, Veprek R G, Witzigmann B and Hangleiter A 2009 Phys. Rev. B 80115320

[23] Hinckley J M and Singh J 1990 Phys. Rev. B 423546

[24] Konar A, Fang T, Sun N and Jena D 2010 Phys. Rev. B 82193301

[25] Konar A, Fang T, Sun N and Jena D 2011 Appl. Phys. Lett. 98022109

[26] Haskell B A et al 2005 J. Electron. Mater. 34357

[27] Hsiao C et al 2010 J. Appl. Phys. 107073502

[28] Ueno K, Kobayashi A, Ohta J and Fujioka H 2010 Japan. J. Appl. Phys. 49060213

[29] McLaurin M, Mates T E, Wu F and Speck J S 2006 J. Appl. Phys. 100063707

[30] McLaurin M and Speck J S 2007 Phys. Status Solidi (RRL) 1110

[31] Baik K H et al 2010 IEEE. Photon. Technol. Lett. 22595

[32] Stampfl C and Van de Walle C G 1998 Phys. Rev. B 24 R15052

[33] Rebane Y T, Shreter Y G and Albrecht M 1997 Phys. Status Solidi a 164141

[34] Griffiths D J 2005 Introduction to Quantum Mechanics (Singapore: Pearson Education)

[35] Erginsoy C 1950 Phys. Rev. 791013

[36] Brooks H 1951 Phys. Rev. 83879

[37] Weimann N G and Eastman L F 1998 J. Appl. Phys. 833656

[38] Lo I et al 2008 Appl. Phys. Lett. 92202106

[39] Zakharov D N and Liliental-Webber Z 2005 Phys. Rev. B 71235334

[40] Seeger K 1999 Semiconductor Physics: An Introduction 7th edn (New York: Springer) p 173

[41] Pödör B 1966 Phys. Status Solidi 16 K167

[42] Look D C and Sizelove J R 1998 Phys. Rev. Lett. 821237
[43] Jena D, Gossard A C and Mishra U K 2000 Appl. Phys. Lett. 761707

[44] Look D C et al 1997 Solid State Commun. 102297

[45] Jonen H et al 2011 Appl. Phys. lett. 99011901

[46] Wang H et al 2004 Appl. Phys. Lett. 84499

[47] Chen C Q et al 2002 Appl. Phys. Lett. 813194

[48] Paskova T et al 2005 J. Cryst. Growth 28155

[49] Hirai A et al 2007 Appl. Phys. Lett. 90121119

[50] Sun Y J et al 2002 J. Appl. Phys. 925714

[51] Brandt O, Sun Y J, Dweritz L and Ploog K H 2004 Physica E 23339

[52] Lymperakis L and Neugebauer J 2009 Phys. Rev. B 79241308

[53] Ferry D K and Goodnick S M 1999 Transport in Nanostructures (New York: Cambridge University Press)

[54] Ridley B K 1999 Electrons and Phonons in Semiconductor Multilayers 1st edn (New York: Cambridge University Press) p 26

[55] Davis J 2006 Physics of Low-Dimensional Semiconductors: An Introduction 6th edn (Cambridge: Cambridge University Press)

[56] Gelmont B L, Shur M and Stroscio M 1995 J. Appl. Phys. 77657

[57] Schliemann J and Loss D 2003 Phys. Rev. B 68165311

[58] Zheng Y et al 2003 J. Phys. Soc. Japan 722568

[59] Schmidt M C, Kim K-C, Farrell R M, Feezell D F, Cohen D A, Saito M, Fujito K, Speck J S, DenBaars S P and Nakamura S 2007 Japan. J. Appl. Phys. 46 L190

[60] Enya Y et al 2009 Appl. Phys. Express 2082101

[61] Dadgar A, Ravash R, Veit P, Schmidt G, Muller M, Dempewolf A, Bertram F, Wieneke M, Christen J and Krost A 2011 Appl. Phys. Lett. 99021905

[62] Massoud H Z 1988 J. Appl. Phys. 632000

[63] Crosslight Software Inc., Burnaby, Canada ApSys http://www.crosslight.com

[64] Simon J, Protasenko V, Lian C, Xing H and Jena D 2010 Science 32760

[65] Verma J, Simon J, Protasenko V, Kosel T, Xing H and Jena D 2011 Appl. Phys. Lett. 99171104

[66] Akyol F, Nath D N, Gu E, Park P S and Rajan S 2011 Japan. J. Appl. Phys. $\mathbf{5 0} 052101$

[67] Kroemer H 1958 Phys. Rev. 1091856

[68] Ridley B K, Schaff W J and Eastman L F 2005 J. Appl. Phys. 97094503

[69] Jena D et al 2011 Phys. Status Solidi a 2081511

[70] Grundmann M J and Mishra U K 2007 Phys. Status Solidi c 42830

[71] Simon J, Zhang Z, Goodman K, Xing H, Kosel T, Fay P and Jena D 2009 Phys. Rev. Lett. 103026801

[72] Krishnamoorthy S, Nath D N, Akyol F, Park P S, Esposto M and Rajan S 2010 Appl. Phys. Lett. 97203502

[73] S-Bhalla G, Bell C, Ravichandran J, Siemons W, Hikita Y, Salahuddin S, Hebard A F, Hwang H Y and Ramesh R 2011 Nat. Phys. 780 